\title{
Stimulus Selectivity and Spatial Coherence of Gamma Components of the Local Field Potential
}

\author{
Xiaoxuan Jia, ${ }^{1}$ Matthew A. Smith, ${ }^{3}$ and Adam Kohn ${ }^{1,2}$ \\ ${ }^{1}$ Dominick Purpura Department of Neuroscience and ${ }^{2}$ Department of Ophthalmology and Vision Sciences, Albert Einstein College of Medicine, Bronx, New \\ York 10461, and ${ }^{3}$ Center for the Neural Basis of Cognition and Department of Neuroscience, University of Pittsburgh, Pittsburgh, Pennsylvania 15213
}

The gamma frequencies of the local field potential (LFP) provide a physiological correlate for numerous perceptual and cognitive phenomena and have been proposed to play a role in cortical function. Understanding the spatial extent of gamma and its relationship to spiking activity is critical for interpreting this signal and elucidating its function, but previous studies have provided widely disparate views of these properties. We addressed these issues by simultaneously recording LFPs and spiking activity using microelectrode arrays implanted in the primary visual cortex of macaque monkeys. We find that the spatial extent of gamma and its relationship to local spiking activity is stimulus dependent. Small gratings, and those masked with noise, induce a broadband increase in spectral power. This signal is tuned similarly to spiking activity and has limited spatial coherence. Large gratings, however, induce a gamma rhythm characterized by a distinctive spectral "bump," which is coherent across widely separated sites. This signal is well tuned, but its stimulus preference is similar across millimeters of cortex. The preference of this global gamma rhythm is sensitive to adaptation, in a manner consistent with its magnifying a bias in the neuronal representation of visual stimuli. Gamma thus arises from two sources that reflect different spatial scales of neural ensemble activity. Our results show that there is not a single, fixed ensemble contributing to gamma and that the selectivity of gamma cannot be used to infer its spatial extent.

\section{Introduction}

Local field potentials (LFPs) reflect coordinated synaptic input and slow intrinsic conductances in neurons (Mitzdorf, 1985; Buzsaki, 2006) and thus provide a potentially useful view of neuronal ensemble activity. Gamma components of the LFP provide a physiological correlate of perceptual and cognitive phenomena (Pesaran et al., 2002; Gail et al., 2004; Wilke et al., 2006; Womelsdorf et al., 2006; Fries et al., 2008) and have been suggested to play an active role in cortical processing.

The spatial extent and functional specificity of gamma are critical constraints on the role it may play in cortical processing. To function as a global reference signal (e.g., an internal clock) (Hopfield, 2004; Fries et al., 2007), gamma would need to form a widespread, coherent rhythm, potentially shared among neuronal ensembles with different response properties. To select specific subsets of neurons [e.g., those representing an attended location (Fries, 2009)], gamma would need to be limited in ex-

\footnotetext{
Received Feb. 5, 2011; revised April 13, 2011; accepted April 29, 2011.

Author contributions: X.J. and A.K. designed research; X.J., A.K., and M.A.S. performed research; X.J. analyzed data; X.J. and A.K. wrote the paper.

This work was supported by National Institutes of Health Grant EY016774 (A.K.) and Grants EY015958 and EY018894 (M.A.S.). We thank Amin Zandvakili, Stephanie Wissig, and Carlyn Patterson for assistance with data collection; Ryan Kelly for technical assistance; and members of the laboratory, Ruben Coen Cagli, Franco Pestilli, Jason Samonds, and Robert Shapley, for comments on an earlier version of this manuscript. We are grateful to Tai Sing Lee in whose laboratory we recorded data from an awake behaving macaque.

The authors declare no competing financial interests.

Correspondence should be addressed to either Xiaoxuan Jia or Adam Kohn, Department of Neuroscience, Kennedy Center, Room 822, Albert Einstein College of Medicine, 1410 Pelham Parkway South, Bronx, NY 10461. E-mail: jxiaoxuan@gmail.com or adam.kohn@einstein.yu.edu.

DOI:10.1523/JNEUROSCI.0645-11.2011

Copyright $\odot 2011$ the authors $\quad 0270-6474 / 11 / 319390-14 \$ 15.00 / 0$
}

tent. To link distributed neurons into an ensemble [e.g., binding or dynamically routing information (Gray, 1999; Buzsaki, 2006; Colgin et al., 2009)], gamma would need to target specific subsets of cells but also be coherent across locations.

The spatial extent and functional specificity of gamma are unclear. Recently, Katzner et al. (2009) showed that the evoked LFP, a stimulus-locked transient response, reflects neural activity within $250 \mu \mathrm{m}$ of the recording site. Xing et al. (2009) showed that this "spatial footprint" reflects the volume conduction of extracellular fields (estimated to be $250 \mu \mathrm{m}$ ) and the extent of the neural ensemble generating the signal. However, these measures of passive propagation and the evoked LFP do not directly address the extent of gamma. This is because gamma is an induced signal-an intrinsic rhythm generated by specific neurons and circuits (for review, see Bartos et al., 2007; Whittington et al., 2011) that are modulated by, but not time locked to, stimulus drive (Kruse and Eckhorn, 1996; Juergens et al., 1999; Brosch et al., 2002; Siegel and König, 2003; Tallon-Baudry, 2003).

Previous studies have provided disparate views of the extent and functional specificity of gamma. In primary visual cortex (V1), gamma is stimulus selective (Gray and Singer, 1989; Frien et al., 2000; Siegel and König, 2003; Henrie and Shapley, 2005; Liu and Newsome, 2006; Berens et al., 2008), suggesting that the relevant circuits have a limited extent: a spatially distributed origin would involve averaging ensembles with different preferences and should thus produce a relatively unselective signal. However, gamma has sometimes (Liu and Newsome, 2006; Katzner et al., 2009), but not always (Kreiman et al., 2006; Berens et al., 2008), been found to have the same preference as spiking activity recorded at the same site. As a result, it has been suggested 
to reflect activity within a few hundred micrometers up to millimeters of the electrode. Another approach to estimating the extent of gamma is to measure it simultaneously at different locations. This has revealed a signal that is coherent across many millimeters of cortex (Juergens et al., 1999; Frien and Eckhorn, 2000; Leopold et al., 2003) and even across regions (Murthy and Fetz, 1992; Pesaran et al., 2002; Schoffelen et al., 2005; Popescu et al., 2009). The presence of gamma in scalp recordings also suggests a widespread coherent signal (Tallon-Baudry, 2003).

To clarify the spatial extent of gamma and its relationship to neuronal activity, we measured both signals simultaneously using multielectrode arrays implanted in the superficial layers of macaque $\mathrm{V} 1$. We compared the response properties of gamma across locations and, at each site, with local spiking activity, for a range of stimulus manipulations.

\section{Materials and Methods}

Animal preparation and electrophysiology. We recorded data from eight anesthetized, adult male macaque monkeys (Macaca fascicularis). The techniques we use have been described in detail previously (Smith and Kohn, 2008). In brief, anesthesia was induced with ketamine (10 mg/kg) and maintained during preparatory surgery with isoflurane $(1.5-2.5 \%$ in $95 \% \mathrm{O}_{2}$ ). Anesthesia during recordings was maintained with sufentanil citrate $\left(6-18 \mu \mathrm{g} \cdot \mathrm{kg}^{-1} \cdot \mathrm{h}^{-1}\right.$, adjusted as needed for each animal). Vecuronium bromide $\left(0.1 \mathrm{mg} \cdot \mathrm{kg}^{-1} \cdot \mathrm{h}^{-1}\right)$ was used to suppress eye movements. Drugs were administered in normosol with dextrose $(2.5 \%)$ to maintain physiological ion balance. Physiological signs (ECG, blood pressure, $\mathrm{SpO}_{2}$, end-tidal $\mathrm{CO}_{2}$, $\mathrm{EEG}$, temperature, and urinary output and osmolarity) were monitored to ensure adequate anesthesia and animal well-being. Temperature was maintained at $36-37 \mathrm{C}^{\circ}$.

Data were also obtained from an awake behaving male rhesus macaque monkey (Macaca mulatta). Detailed methods regarding our procedures for training and recording from awake behaving macaques can be found in a previous publication (Smith et al., 2007). All procedures were approved by the Institutional Animal Care and Use Committee of Carnegie Mellon University (awake recordings only) and the Albert Einstein College of Medicine at Yeshiva University (anesthetized recordings only) and were in compliance with the guidelines set forth in the United States Public Health Service Guide for the Care and Use of Laboratory Animals.

We implanted a $4 \times 4 \mathrm{~mm}$ multielectrode array $(0.4 \mathrm{~mm}$ spacing and $1 \mathrm{~mm}$ electrode length) with 100 electrodes into the upper layers of primary visual cortex $(\sim 0.6-0.8 \mathrm{~mm}$ deep in anesthetized animals; 1 $\mathrm{mm}$ for awake recordings), $\sim 10 \mathrm{~mm}$ lateral to the midline and $\sim 8 \mathrm{~mm}$ posterior to the lunate sulcus. Two reference wires were placed between the brain surface and the dura. Events larger than a user-defined threshold were recorded (Cyberkinetics Neurotechnology Systems). We applied additional voltage thresholding off-line (Plexon Offline Sorter) to remove any remaining noise. Units from the same electrode were then combined to form multiunit activity (MUA). The peak firing rate of this signal was on average $20.7 \pm 0.5 \mathrm{ips}$, suggesting it arose from a handful of neurons at most. LFPs were obtained by bandpass filtering the same signal between 0.3 and $250 \mathrm{~Hz}$ and sampling at 1 or $2 \mathrm{kHz}$.

In some experiments, a separate linearly arranged multielectrode device (Thomas Recording) was positioned between the lunate sulcus and the array, with each electrode referenced to the guide tubes. Raw signals recorded from this device were bandpass filtered between 0.5 and $250 \mathrm{~Hz}$, and digitized at $1 \mathrm{kHz}$. To remove $60 \mathrm{~Hz}$ noise, we applied a fourth-order Butterworth band-stop filter to the raw data.

Visual stimulation. Visual stimuli were generated using custom software (EXPO or Matlab Psychtoolbox) and displayed on a monitor with a resolution of $1024 \times 768$ pixels, viewed at a distance of $110 \mathrm{~cm}(58 \mathrm{~cm}$ for awake recordings). We mapped the spatial receptive field of each channel by presenting small, drifting gratings $\left(0.6^{\circ} ; 250 \mathrm{~ms}\right.$ duration $)$ at a range of spatial positions. We centered our stimuli on the aggregate receptive field of the recorded units. Stimuli were viewed binocularly and presented for $1 \mathrm{~s}$ at full contrast. We presented each stimulus 25 times (100 times for measurements of dynamics), in a pseudorandom sequence. In awake
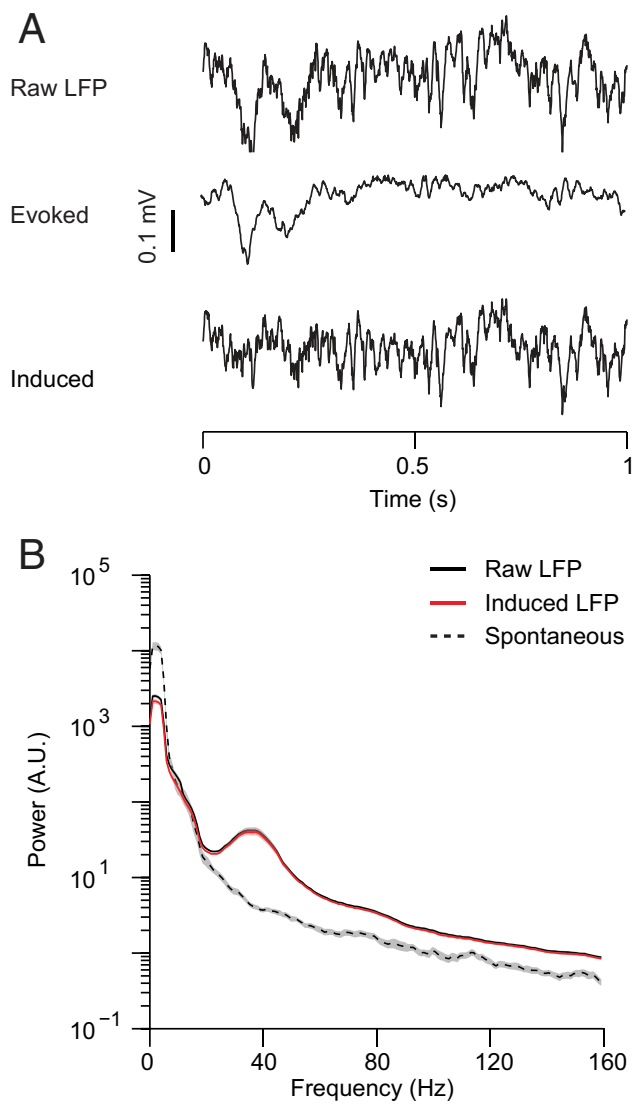

Figure 1. Evoked and induced components of the LFP. $A$, Single epoch examples of the raw LFP (top) and the induced component (bottom). The induced component is calculated by subtracting the evoked component (middle) from the raw signal. $\boldsymbol{B}$, Example of the power spectrum of the induced LFP (red), compared with the raw LFP (black). Spontaneous activity was measured with a uniform luminance screen (gray dashed).

recordings, the animal was required to maintain fixation within a $1^{\circ}$ window during the $1 \mathrm{~s}$ stimulus presentation and to make a saccade to a random target location at stimulus offset.

For our measurements of tuning at different sizes, stimuli were viewed monocularly. In these experiments, we included only those sites whose receptive field center was within $0.5^{\circ}$ of the stimulus center. This yielded typically approximately one-half of the recorded sites, with a maximal separation of $3.57 \pm 0.02 \mathrm{~mm}$ on average. In our noise-masking experiments, spatial noise was created by selecting small patches $\left(0.06 \times 0.06^{\circ}\right)$ randomly in the original image, computing the mean luminance of each patch, and then randomly permutating the patches (Zhou et al., 2008). The noise level $(20,50$, and $80 \%)$ was defined by the proportion of total area replaced by noise. Noise was randomly distributed on each frame.

Data analysis. We isolated the induced components of the LFP by subtracting the evoked signal (the average response across trials, for each stimulus) from the raw signal on each trial (Fig. $1 A$ ). We then analyzed responses $100-1000 \mathrm{~ms}$ after stimulus onset, when the magnitude of the evoked component was minimal. We did this to separate these two different components of the LFP (Katzner et al., 2009), but the power spectra of the raw LFPs (i.e., not subtracting the evoked component) were in fact similar to the induced LFPs, except at low frequencies $(<20 \mathrm{~Hz})$ (Fig. $1 B)$. All of the findings reported here were thus similar when based on raw responses, even when we included the onset transient.

We calculated the power spectrum with a multitaper method (Mitra and Pesaran, 1999), which uses a set of orthogonal Slepian tapers to provide a good estimate of power for a limited number of trials and small time windows. For a signal of duration $T$ and a desired half-bandwidth of $W$ (determining the smoothness of the spectrum), the taper number is given by $k=2 T W-1$. For most of our analysis, the duration of the analyzed epoch was $0.9 \mathrm{~s}$, so we used eight tapers to provide a half- 
bandwidth equal to $5 \mathrm{~Hz}$. For the analysis of dynamics, we used a $129 \mathrm{~ms}$ sliding window ( $25 \mathrm{~ms}$ steps) and three tapers.

Tuning of the LFP was based on its average power within $4 \mathrm{~Hz}$ bins, spanning the range from 0 to $160 \mathrm{~Hz}$. Orientation preference was determined by the vector sum of responses to 16 test directions $\left(22.5^{\circ}\right.$ step). An orientation selectivity index (OSI) was calculated as the vector sum of the response vectors (combining responses to different drift directions of the same orientation), normalized by the sum of lengths of the vectors (Leventhal et al., 1995). For computing the OSI, we defined response strength with respect to that driven (MUA) or induced (LFP) by the least preferred orientation. Using spontaneous activity as a baseline was precluded because its power could be either greater or less than the stimulusinduced power, depending on the frequency band of interest.

Only sites with an OSI $>0.2$ for both MUA and gamma were kept for tuning-related analysis (i.e., for determining the orientation preference of the site or for the correlation between tuning curves). To determine the preference of individual sites, we used the best tuned frequency band [i.e., the $4 \mathrm{~Hz}$ band with highest selectivity (Berens et al., 2008)] in the gamma range $(30-50 \mathrm{~Hz})$.

We quantified the variance of orientation preferences in the population, $\sigma^{2}$, as follows:

$$
1-\frac{\sqrt{\left(\sum_{i=1}^{n} \cos \left(2 \theta_{i}\right)\right)^{2}+\left(\sum_{i=1}^{n} \sin \left(2 \theta_{i}\right)\right)^{2}}}{n},
$$

where $\theta_{i}$ is the orientation preference for channel $i$ and $n$ is the number of sites.

To determine the tuning similarity between MUA and LFP from the same channel ( $r_{\text {MUA-LFP }}$ or $r_{\text {MUA- } \gamma}$ when comparing only to the gamma components) or between two LFP sites $\left(r_{\text {LFP-LFP }}\right.$ or $\left.r_{\gamma-\gamma}\right)$, we calculated the Pearson correlation between their tuning. We found that the peak gamma frequency was lower for large gratings (Gieselmann et al., 2008) and for stimuli masked by noise [similar to the effect of lowering stimulus contrast (Ray and Maunsell, 2010)]. To enable a meaningful comparison across conditions, we therefore computed tuning correlations (and all other measures, except orientation preferences) based on the average gamma power in $30-50 \mathrm{~Hz}$. We obtained similar results if our analysis was based on any subband in this frequency range.

To measure the spatial coherence of the LFP, we calculated the coherency between signals measured at different sites ( $x$ and $y$ ) as follows:

$$
C_{x y}(f)=\frac{S_{x y}(f)}{\sqrt{S_{x x}(f) S_{y y}(f)}},
$$

where $S_{x y}$ is the cross-spectrum calculated with the multitaper method (duration of $0.9 \mathrm{~s}$, half-bandwidth of $5 \mathrm{~Hz}$, and taper number of 8), and $S_{x x}$ and $S_{y y}$ are the respective autospectra (Pesaran et al., 2002). $C_{x y}$ is a complex number. Its modulus is the coherence (ranging from 0 to 1 ), a measure of the relationship between two signals as a function of frequency $(f)$. The phase of $C_{x y}$ is the relative phase difference between the two signals, as a function of frequency.

All indications of variation in the graphs and text are SEMs. The statistical significance of all results was evaluated with two-tailed $t$ tests, unless otherwise noted. Significance of correlation values was assessed after applying the Fisher $Z$-transform to the data.

\section{Results}

We implanted microelectrode arrays in the upper layers of $\mathrm{V} 1$ of eight anesthetized macaque monkeys and recorded spiking activity and LFPs $(0.3-250 \mathrm{~Hz})$ simultaneously on each electrode. Each array covered roughly a $4 \times 4 \mathrm{~mm}$ cortical region, corresponding to the representation of $\sim 2-3^{\circ}$ of the lower visual field $\left(2-5^{\circ}\right.$ from the fovea).

\section{Orientation tuning of gamma power}

To compare the tuning of gamma power to local spiking activity, we measured responses to large gratings $\left(7.4\right.$ or $10^{\circ}$ in diameter) drifting in 16 different directions. Gratings had a spatial frequency $(1 \mathrm{cycle} / \mathrm{deg})$ and drift rate $(6.25 \mathrm{~Hz})$ chosen to evoke robust activity in parafoveal V1 (Foster et al., 1985). Spiking activity was isolated from the filtered voltage signal $(250 \mathrm{~Hz}-10$ $\mathrm{kHz}$ ) with a user-defined threshold and sorted off-line to yield MUA. Tuning of the LFP was generated by computing the power spectrum of the induced signal on each trial and measuring how power in gamma and other frequency bands depended on stimulus orientation. Consistent with previous studies, we found that the LFP was stimulus selective. An example of its tuning at one site (for frequencies between 32 and $36 \mathrm{~Hz}$ ) is shown in Figure $2 A$, together with that of the local MUA.

We quantified tuning quality using a selectivity index, for which a value of 0 indicates an equal response to all orientations and a value of 1 indicates an elevated response to a single orientation, relative to all others. The low-frequency components of the LFP were poorly tuned, but for frequencies in the gamma range and higher (above $\sim 30 \mathrm{~Hz}$ ) selectivity was relatively high (Fig. $2 B$, black line) ( $n=680$ sites in 8 implants), albeit lower than that of MUA $(0.47 \pm 0.01$; indicated with black dot to the right).

To quantify the similarity of LFP orientation tuning to local MUA, we computed the correlation between their tuning at each site (termed $r_{\text {MUA-LFP }}$ ). A value of $r_{\text {MUA-LFP }}$ near 1 indicates very similar tuning; a value near -1 would indicate the opposite. For the tuning curves in Figure $2 A, r_{\text {MUA-LFP }}$ was 0.22 . Consistent with previous V1 studies (Frien et al., 2000; Siegel and König, 2003; Berens et al., 2008), we found that high-frequency components of the LFP were more similarly tuned to local spiking activity than low-frequency components (Fig. 2B, red trace). However, despite the tendency for $r_{\text {MUA-LFP }}$ to increase for higher frequencies, we observed a clear deviation in the range of 30 to $50 \mathrm{~Hz}$ (low gamma frequencies, hereafter "gamma"; indicated by box): tuning of gamma was relatively distinct from that of MUA. A similar effect was observed at harmonics of these frequencies $(70-90 \mathrm{~Hz})$ in some, but not all, implants.

To explore this further, we compared the preferred orientation of gamma and MUA at each tuned site (selectivity index, $\geq 0.2$ ). The two signals had a similar preference at some sites but not at others (Fig. $2 C$ for data from an example implant). Strikingly, this occurred because the orientation preference of gamma was often similar across sites. This is evident in the marginal histograms (Fig. 2C), which show a roughly uniform distribution of preferences for spikes (circular variance, $\sigma^{2}$, of 0.73 ) and a clearly biased distribution for gamma $\left(\sigma^{2}\right.$ of 0.23$)$, with a preference of $\sim 110^{\circ}$ being the most common. We found a similar pattern in other implants: the distribution of preferences was consistently uniform for spikes ( $\sigma^{2}$ of $0.75 \pm 0.03 ; n=8$ ) but strongly biased for gamma $(0.23 \pm 0.06$; $p \ll 0.001$ for the difference between the two signals; Wilcoxon's rank sum test). The orientation preferred by gamma, however, was different in each implant.

We considered that our results might be due to anesthesia, so we recorded responses using the same type of microelectrode arrays and visual stimuli in an awake monkey (see Materials and Methods). The data are consistent with, but more striking than, those observed in anesthetized animals (Fig. 2D). In this data set, the orientation preference of gamma was always near $60^{\circ}\left(\sigma^{2}\right.$ of 0.07 ), whereas simultaneously recorded MUA showed a wide range of preferences ( $\sigma^{2}$ of 0.80$)$.

To evaluate the cortical distance over which orientation tuning is similar for gamma and other frequency components, we computed the correlation between its tuning $\left(r_{\text {LFP-LFP }}\right)$ at all se- 
A

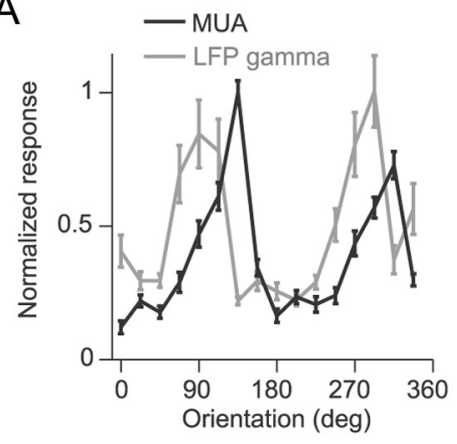

C

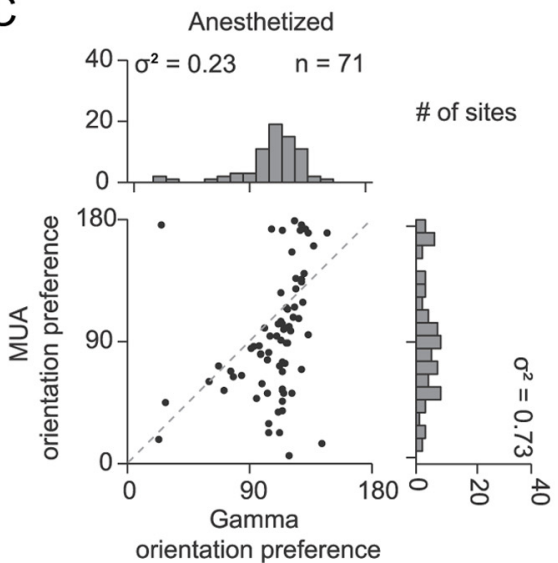

$\mathrm{B}$

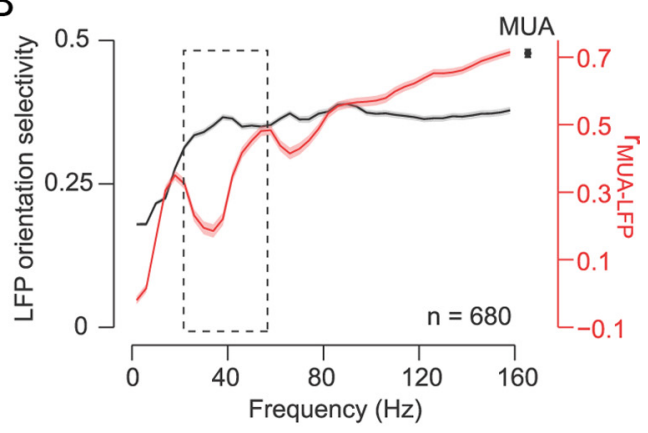

D

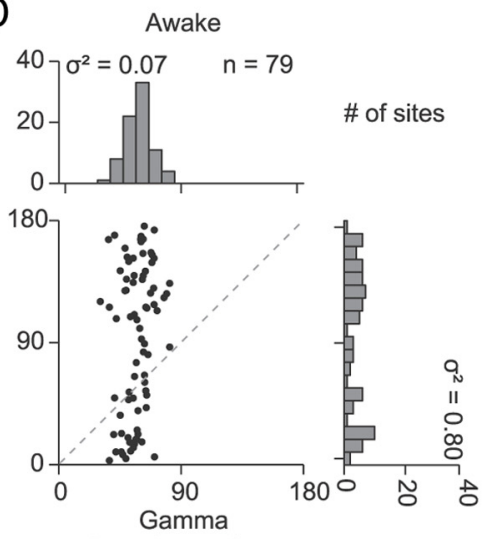

orientation preference

Figure 2. Comparison of the orientation tuning of the LFP with local spiking activity. $A$, Example tuning curves for MUA (black) and gamma power (gray) at the same site ( $n=25$ repeats). $\boldsymbol{B}$, Population average trends for LFP orientation selectivity (black) and correlation between the tuning of MUA and the LFP ( $r_{\text {MUA-LFP }}$; red), as a function of frequency ( $n=680$ sites). The mean orientation selectivity index for MUA is indicated to the right side (black dot). $C$, Comparison of orientation preferences for gamma and MUA ( $n=71$ sites from one implant). The circular variance $\left(\sigma^{2}\right)$ of the distributions was 0.23 for gamma and 0.73 for MUA. $D$, Similar example for data from an awake monkey ( $n=79$ sites).
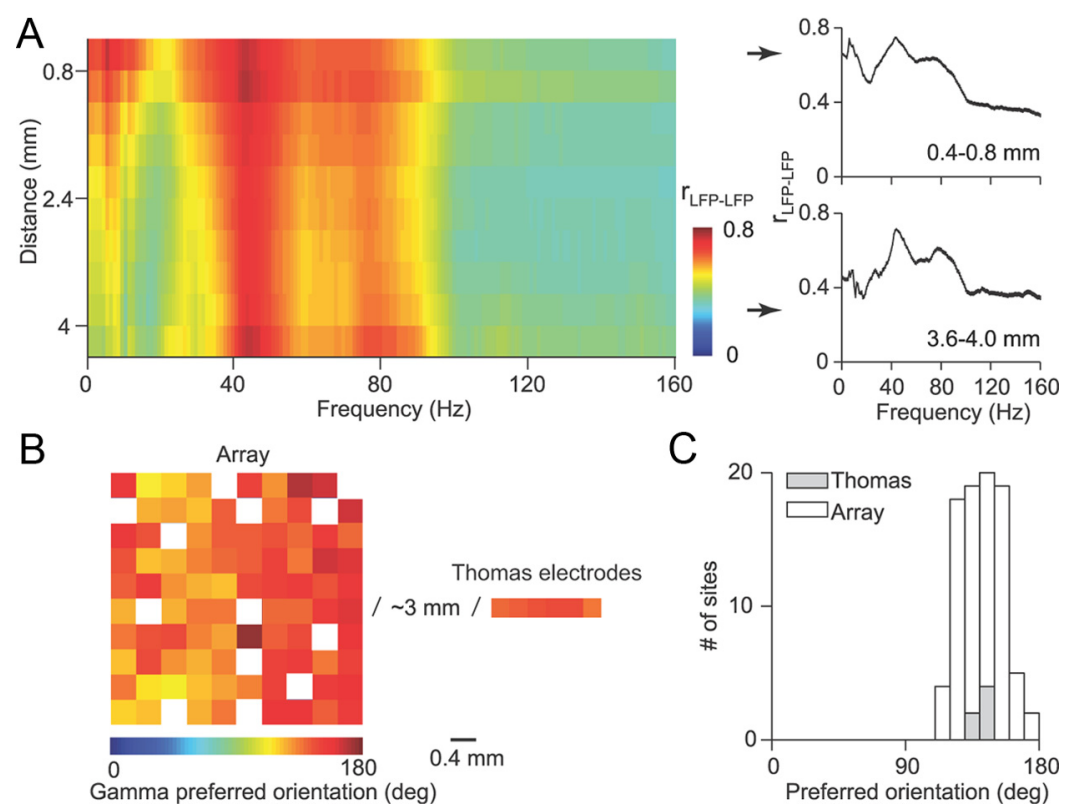

C

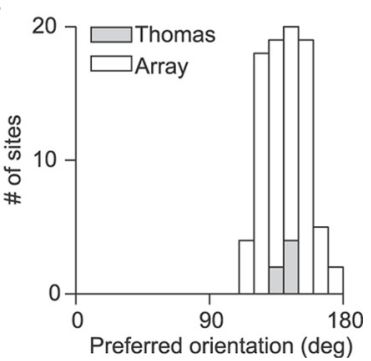

Figure 3. Relationship between orientation tuning of the LFPs measured at different sites. $\boldsymbol{A}$, Population average of $r_{\text {LFP-LFP }}$ as a function of distance, for sites with orientation selectivity $\geq 0.2$. Cross sections of the color plot for distances of $0.4-0.8 \mathrm{~mm}(n=2233$ pairs) and $3.6-4.0 \mathrm{~mm}(n=1347$ pairs) are shown on the right. $\boldsymbol{B}$, An example of orientation preference in the gamma band, from simultaneous recordings using two multielectrode systems. Each square represents the orientation preference of one recording site, plotted according to its spatial position. C, Distributions of orientation preference for the example in $\boldsymbol{B}$.

lective sites and sorted the data according to the distance between electrodes (Fig. $3 A)(n=31,211$ pairs of sites). The value of $r_{\text {LFP-LFP }}$ was well maintained across a distance of $>4 \mathrm{~mm}$ for frequencies between $\sim 35$ and $90 \mathrm{~Hz}$. In the $30-50 \mathrm{~Hz}$ band, it decayed only $10.2 \%$, from $0.69 \pm$ 0.01 for nearby sites $(0.4-0.8 \mathrm{~mm} ; n=$ $2233)$ to $0.62 \pm 0.01$ at distances of $4-5$ $\mathrm{mm}(n=571)$. At lower frequencies $(<30$ $\mathrm{Hz}), r_{\text {LFP-LFP }}$ decayed more rapidly ( $35 \%$ over the same distance). At higher frequencies $(>100 \mathrm{~Hz}), r_{\text {LFP-LFP }}$ was significantly smaller, even for nearby electrodes, as each site had a different preference.

Because gamma tuning was similar across the entire spatial extent of the array, we performed simultaneous recordings with a separate multielectrode device (Thomas Recording) positioned $\sim 3 \mathrm{~mm}$ away (five penetrations in four monkeys). These electrodes were arranged linearly and oriented orthogonally to the nearest edge of the array, providing neurons that were $3-9 \mathrm{~mm}$ from those sampled by the array. We used large gratings covering the receptive fields of all neurons to induce gamma activity. The tuning measured at these additional sites had a similar preference to those measured by the array (example shown in Fig. $3 B$ with each square representing the preference at one recording site and the respective distributions shown in Fig. $3 C$ ), with a difference in mean preference of $4.5 \pm 1.0^{\circ}$. The orientation preference of gamma can thus be shared over many millimeters of cortex. Note that these recordings used independent multielectrode systems, with distinct electrical references (see Materials and Methods). As a result, these observations preclude the possibility that shared tuning involved an array-specific artifact (e.g., cross talk between electrodes) or arose from a common referencing of array electrodes (for related findings and further discussion, see also Berens et al., 2008).

Our results show that, when gamma is induced by large gratings, it is orientation selective but with a preference distinct from local spiking activity and similar across millimeters of cortex. Since its tuning is distinct, its power is thus not indicative of the strength of activity in local ensembles.

\section{Dependence of gamma tuning on stimulus size}

The similar tuning of gamma we observed across sites was based on responses to large gratings. Previous studies have shown that gamma power is weakened by reducing stimulus size (Bauer et al., 1995; Gie- 
selmann and Thiele, 2008). We therefore tested how this manipulation affects the similar tuning of gamma across sites and the relationship between its tuning and that of local spiking activity.

To determine the influence of stimulus size, we measured orientation tuning with gratings ranging from 1 to $10^{\circ}$ in diameter. Stimuli were centered on the aggregate receptive field of the MUA, as determined by a separate mapping procedure (see Materials and Methods). We analyzed only those sites driven by our smallest stimulus so that the same locations would be compared across conditions. Consistent with previous observations (Bauer et al., 1995; Gieselmann and Thiele, 2008), gamma power increased with stimulus size (Fig. $4 A$ ), nearly doubling over the range measured. In contrast, the spiking activity at these sites was suppressed by stimuli $>1^{\circ}$ (Fig. $4 A$ ), as expected given the prevalence of surround suppression in V1 (Angelucci and Bressloff, 2006).

We found that the relationship between the orientation tuning of MUA and gamma depended strongly on stimulus size. When driven with small gratings $\left(1^{\circ}\right)$, gamma power had a similar preference to MUA at all sites, as shown in Figure $4 B$ for a single implant. When stimulated with larger gratings $\left(10^{\circ}\right)$, the preference of gamma changed: it became more similarly tuned across sites, and its preference at many sites became distinct from the local MUA (Fig. 4C). Across our data set, we found $r_{\gamma-\gamma}$, the tuning similarity of gamma, increased strongly with stimulus size (Fig. 4D), from $0.11 \pm 0.01$ to $0.50 \pm 0.01(n=2961$ pairs of sites in 3 implants). The similarity between the tuning of MUA and gamma $\left(r_{\text {MUA- } \gamma}\right)$, however, decreased from $0.42 \pm 0.03$ to $0.25 \pm$ $0.03(n=129$ sites $)$, indicating these two signals became more distinct for large stimuli. Although the preference of gamma changed dramatically with stimulus size, its selectivity did not: the mean selectivity for small stimuli was $0.31 \pm 0.01 \mathrm{com}-$ pared with $0.37 \pm 0.01$ for large gratings, a small but statistically significant increase in tuning quality for the more global signal $(p=0.007)$.

Our results show that gamma has a different preference for small stimuli (i.e., one matched to local spiking activity) and large ones (i.e., one shared across sites). This suggests that gamma does not reflect ensemble activity of a fixed extent. The shared preference we observed across millimeters of cortex, when gamma is induced by large stimuli, suggests a spatially extensive mechanism underlying the signal. For small stimuli, the close match between the preference of spiking activity and gamma measured at the same site strongly suggests a local basis for the signal. Note that small gratings activated $\sim 10 \mathrm{~mm}^{2}$ of cortex, but the preference of gamma matched the spiking activity at each measured location. Thus, the close match between the preferences of these two signals is not a trivial consequence of small gratings only driving circuits near a particular electrode.

\section{Two components of gamma power}

The change in the tuning of gamma with stimulus size was paralleled by a change in the form of the LFP spectra. Small gratings induced a broadband $(20-160 \mathrm{~Hz})$ increase in power, which was stronger for some orientations than others (Fig. 5A). The preference for gamma frequencies was similar to that of both higher $(>50 \mathrm{~Hz})$ and nearby lower $(20-30 \mathrm{~Hz})$ frequencies. Large gratings induced a notable increase in power at gamma frequencies, which strongly exceeded the power of both high and lower (20-30 Hz) frequencies (Fig. 5B) (Gieselmann et al., 2008; Ray and Maunsell, 2010). The tuning of this gamma "bump" was

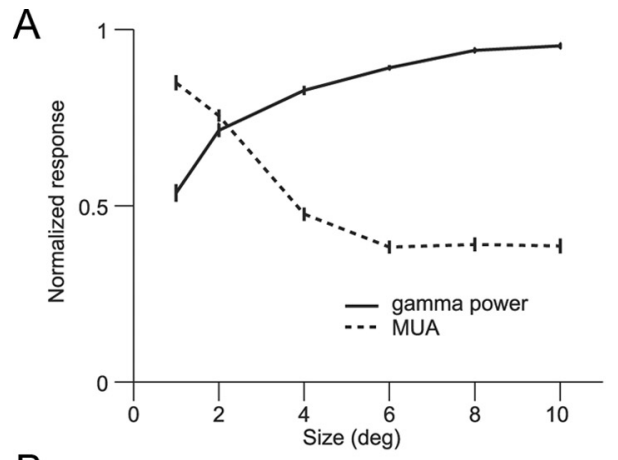

B
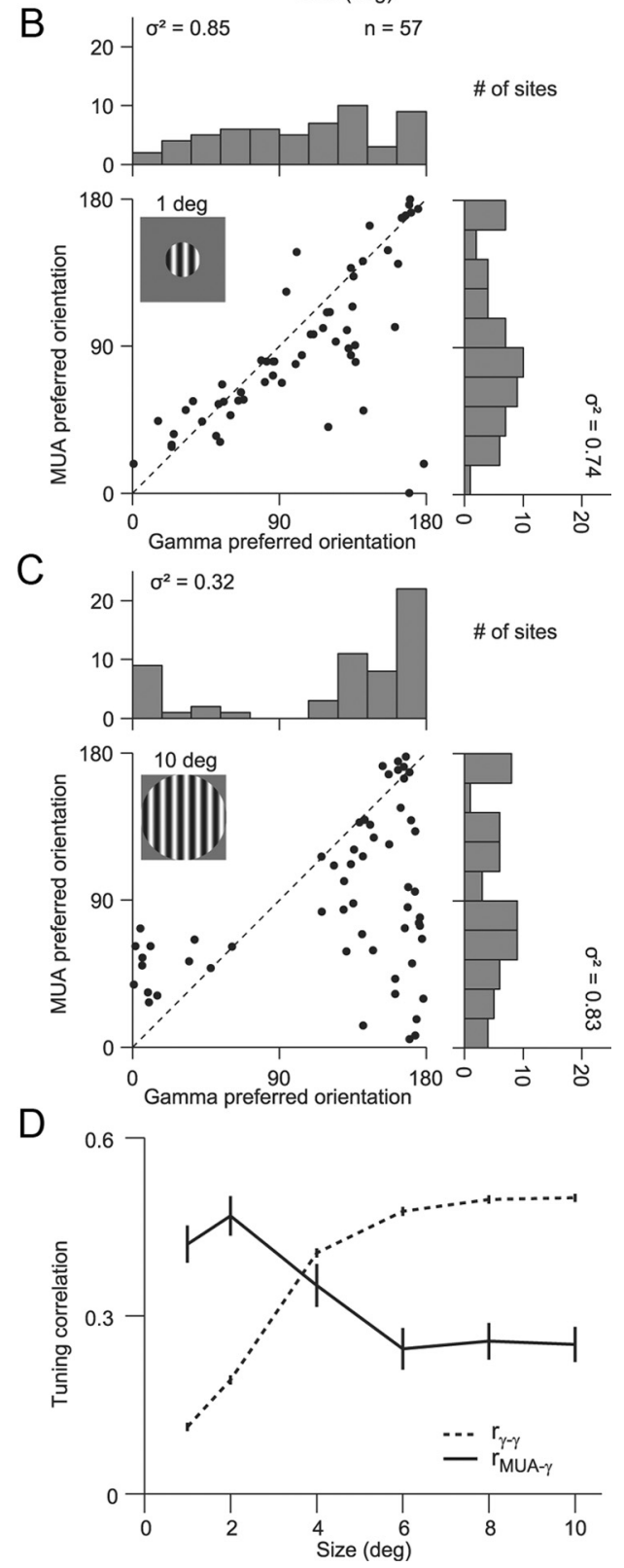

Figure 4. Effect of stimulus size on gamma power and its tuning. $\boldsymbol{A}$, Normalized firing rate (dashed trace) and gamma power (solid black trace) as a function of stimulus size. Data are shown only for sites driven by the smallest stimulus ( $n=129$ sites). $\boldsymbol{B}$, Comparison of orientation preference for activity driven by a $1^{\circ}$ grating ( $n=57$ sites), for a single implant. The preference of gamma was similar to the MUA at all sites, and the distribution of preferences had a similar variance $(0.85$ and 0.74 , respectively). $\boldsymbol{C}$, Orientation preferences for the same sites as in $\boldsymbol{B}$, but when stimulated with a large grating $\left(10^{\circ}\right)$. Gamma preferences dissociated from MUA and became more uniformly tuned, with circular variance equal to 0.32. D, Dependence of $r_{\text {MUA- } \gamma}$ (solid trace; $n=129$ sites) and $r_{\gamma-\gamma}$ (dashed trace; $n=2961$ pairs) on stimulus size. 

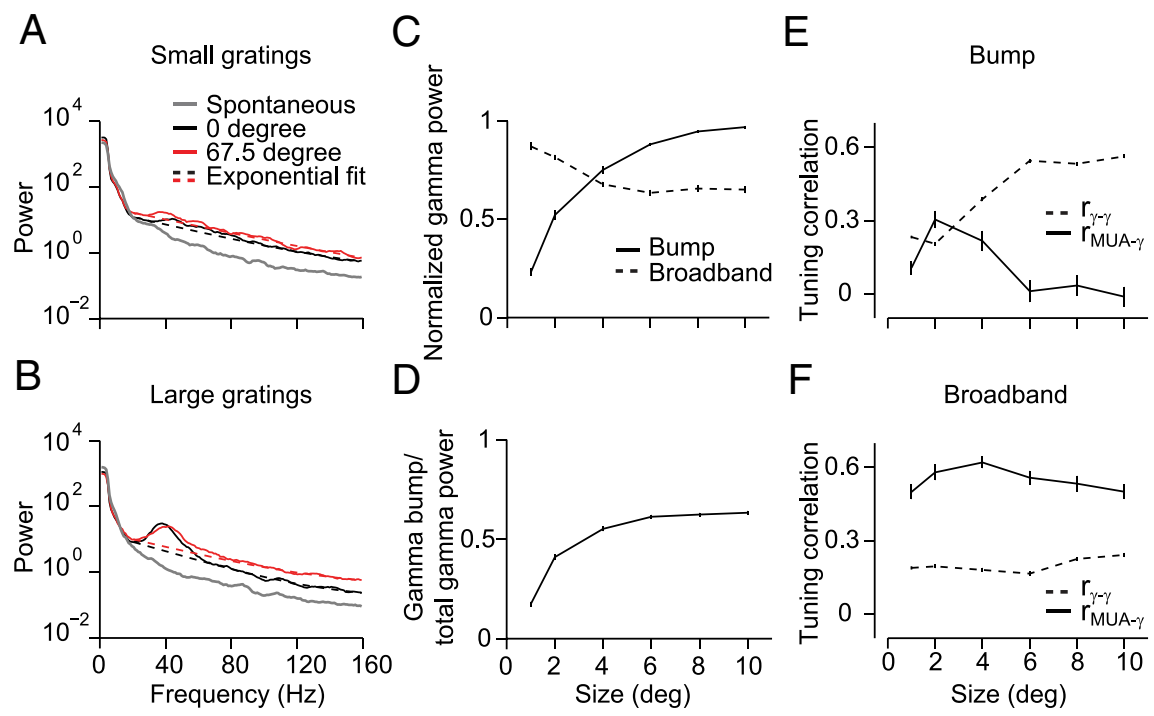

Figure 5. Two components of gamma power and their dependence on stimulus size. $\boldsymbol{A}, \boldsymbol{B}$, Power spectra of LFPs from one recording site, for large $\left(10^{\circ} ; \boldsymbol{A}\right)$ and small $\left(1^{\circ} ; \boldsymbol{B}\right)$ stimuli for two orientations (red and black lines) and spontaneous activity (gray lines). Estimate of broadband component of gamma power is provided by the exponential fit indicated with dashed lines. $C$, Normalized power of the gamma bump and broadband component, as a function of stimulus size ( $n=129$ sites). $\boldsymbol{D}$, Proportion of total power attributable to the gamma bump. $\boldsymbol{E}, \boldsymbol{F}$, Dependence of $r_{\text {MUA- } \gamma}$ (solid trace; $n=129$ sites) and $r_{\gamma-\gamma}$ (dashed trace; $n=$ 2961 pairs), for the gamma bump $(\boldsymbol{E})$ and broadband component $(\boldsymbol{F})$, on stimulus size.

often distinct from neighboring frequencies, suggesting a distinct origin.

We estimated the strength of the broadband component of gamma using an exponential fit to the power measured between $20-26$ and $80-160 \mathrm{~Hz}$ (outside the range of gamma) (Fig. 5A, $B$, dashed lines). For activity induced by small stimuli, this fit captured $92 \pm 2 \%$ of the variance in the spectra over the $20-160 \mathrm{~Hz}$ range. The gamma power estimated by this fit was $87 \pm 1 \%$ of that measured, and it was strongly correlated with the measured gamma across orientations $(r=0.64 \pm 0.02)$. The remaining power-that exceeding this prediction-we refer to as the gamma bump. The broadband component of gamma decreased for larger stimuli (Fig. 5C), much like spiking activity (Fig. 4A); the gamma bump, however, grew with stimulus size (Fig. $5 C$ ). The proportion of total gamma power attributable to the bump thus increased with stimulus size (Fig. 5D).

To compare the tuning of these two components of gamma and their relationship to local spiking activity, we calculated $r_{\text {MUA- } \gamma}$ and $r_{\gamma-\gamma}$ separately for each component (Fig. $5 E, F$ ). We found that the gamma bump for large stimuli (for which it was most accurately measured) had a similar preference across sites $\left(r_{\gamma-\gamma}\right.$ of $\left.0.56 \pm 0.007\right)$ and thus a distinct preference from local spiking activity $\left(r_{\mathrm{MUA}-\gamma}\right.$ of $\left.-0.01 \pm 0.04\right)$. The broadband component of gamma, however, was always similarly tuned to local spiking activity ( $r_{\text {MUA- } \gamma}$ ranging 0.50 to 0.62$)$ and thus tuning across sites was only weakly correlated $\left(r_{\gamma-\gamma}\right.$ ranging from 0.18 to 0.24$)$.

We conclude that there is a component of gamma that arises from broadband changes in power. This component behaves similarly to local spiking activity. Large stimuli induce a second component, which has a shared preference across millimeters of cortex. The flexible relationship between the tuning of gamma and local spiking activity, revealed by changes in stimulus size, can be explained by the relative contribution of these two components to total gamma power.

\section{Manipulations that do not alter stimulus size can also change the preference of gamma}

Manipulating stimulus size shows that the preference of gamma and its relationship to local spiking activity are flexible, but leaves unclear when gamma is likely to display a shared preference across sites. It could be that shared tuning involves mechanisms that are recruited whenever an extensive region of cortex is visually driven, as with large gratings. Alternatively, shared tuning may occur because of the gamma bump induced by such stimuli. If so, manipulations that reduce the power of this component-without altering the extent of visually driven cortexwould be expected to result in tuning similar to that of local multiunit activity.

To distinguish between these possibilities, we manipulated gamma power by masking large gratings with noise (Zhou et al., 2008). This manipulation had a limited effect on V1 firing rate, with the response evoked by unmasked gratings and those masked with $80 \%$ noise being nearly indistinguishable in strength (Fig. 6A, dashed trace): the mean firing rate across stimuli was $8.9 \pm 0.7 \mathrm{spikes} / \mathrm{s}$ for unperturbed grating compared with $8.4 \pm 0.7$ for those masked with $80 \%$ noise $(n=248$ sites in 3 implants). With high levels of noise, however, gamma power was reduced more than twofold (Fig. 6A) (from a normalized value of $0.99 \pm 3 \mathrm{E}-4$ to $0.42 \pm 5 \mathrm{E}-3 ; n=248$ sites) (for related findings, see Lima et al., 2010). This was due primarily to a loss of the gamma bump, as evident in the small proportion of the total power provided by this component with high masking noise (Fig. 6B).

The relationship between the tuning of gamma and local spiking activity depended on the strength of masking noise. Figure $6 \mathrm{C}$ shows the orientation preference for one array at three noise levels. For unmasked gratings ( $0 \%$ noise; top panel), the preference of gamma was similar across sites $\left(\sigma^{2}\right.$ of 0.12 and $r_{\gamma-\gamma}$ of $0.67)$ and distinct from local multiunit activity $\left(r_{\text {MUA- } \gamma}\right.$ of 0.15$)$. With high masking noise ( $80 \%$ noise; bottom panel), the tuning of the two signals became more similar $\left(r_{\text {MUA- } \gamma}\right.$ of 0.56$)$, and, thus, the preference of gamma was no longer shared across sites $\left(\sigma^{2}\right.$ of 0.59 and $r_{\gamma-\gamma}$ of 0.33 ). Across implants, as masking noise was increased, $r_{\gamma-\gamma}$ decreased from $0.74 \pm 3 \mathrm{E}-3$ to $0.48 \pm 2 \mathrm{E}-3$; $p \ll 0.001 ; n=8911$ pairs of sites) and gamma became more similarly tuned to the local MUA ( $r_{\text {MUA- } \gamma}$ increased from $0.14 \pm$ 0.02 to $0.46 \pm 0.02 ; \mathrm{p} \ll 0.001 ; n=228$ sites $)$.

These results show that noise masking disrupts the gamma bump induced by large stimuli and results in gamma whose tuning is similar to local spiking activity. Compared with manipulations of stimulus size, masking required a larger decrease in gamma power to generate a signal with similar preference to local spiking activity: it is only at the highest level of masking noise, when the gamma bump is nearly entirely suppressed (Fig. 6B), that the two signals become more similarly tuned. Thus, while the trends for size and noise masking manipulations are similar, the point at which gamma switches from a shared preference to a local one is different. This is presumably because of numerous differences in the drive provided by these two stimuli. Neverthe- 

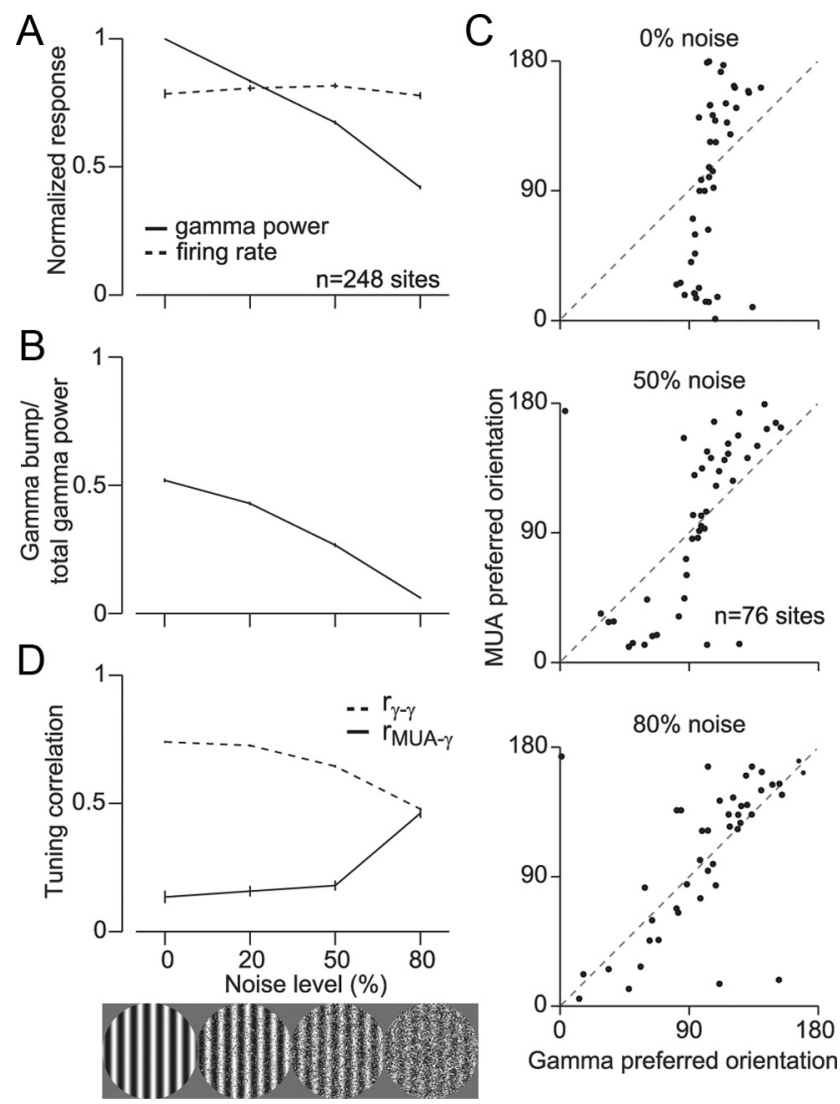

Figure 6. Masking noise modulates gamma power and its tuning. $A$, Effect of masking noise on normalized gamma power (solid trace; $n=248$ sites) and firing rate (dashed trace). $\boldsymbol{B}$, Proportion of total power attributable to the gamma bump. $\boldsymbol{C}$, Comparison of orientation preference of gamma and MUA for three noise levels ( $n=76$ sites in one array). Gamma is more dissociated from local spiking activity when the gamma bump is more prominent. $\boldsymbol{D}$, Dependence of $r_{\text {MUA- } \gamma}$ (solid trace; $n=228$ sites) and $r_{\gamma-\gamma}$ (dashed trace; $n=8911$ pairs; error bars are smaller than the line thickness) on the amount of masking noise. Stimuli size is $10^{\circ}$. Representation of stimuli is shown at the bottom.

less, our masking results clearly show that the change in the tuning of gamma does not require reducing stimulus size or the spatial extent of activated cortex.

To test further the flexible relationship between the tuning of gamma and local spiking activity, we made use of the slow buildup of induced gamma after stimulus onset (Bauer et al., 1995; Ray and Maunsell, 2010). We analyzed responses to large gratings drifting in different directions, with interleaved blank stimuli, using a sliding window (129 ms epoch length with $25 \mathrm{~ms}$ steps). Gamma power increased soon after stimulus onset (Fig. $7 A$, vertical dashed line) but reached its maximum $\sim 250$ ms later, on average ( $n=683$ sites). The proportion of power attributable to the gamma bump reached its maximum at a similar time, but its onset was delayed, revealing that the initial enhancement of gamma reflects a broadband increase in power (Fig. $7 B$ ).

During the initial epoch of the response (25-154 ms after stimulus onset) (Fig. 7C, top), gamma tuning was well matched to the local spiking activity $\left(r_{\text {MUA- } \gamma}\right.$ of 0.47$)$ and had a wide range of preferences $\left(\sigma^{2}\right.$ of 0.87$)$. In later epochs, gamma tuning changed to a common preference across sites ( $\sigma^{2}$ of 0.25 for the epoch from 350 to $479 \mathrm{~ms}$ ). Across implants, we found that $r_{\gamma-\gamma}$ increased markedly over the first few hundred milliseconds of response (from $0.47 \pm 2 \mathrm{E}-3$ to $0.71 \pm 2 \mathrm{E}-3 ; p \ll 0.001$ ) (Fig. $7 D$, gray line). Over the same period, $r_{\text {MUA- } \gamma}$ fell more than twofold (from $0.35 \pm 0.01$ to $0.14 \pm 0.02 ; p \ll 0.001$ ) (Fig. $7 D$, black
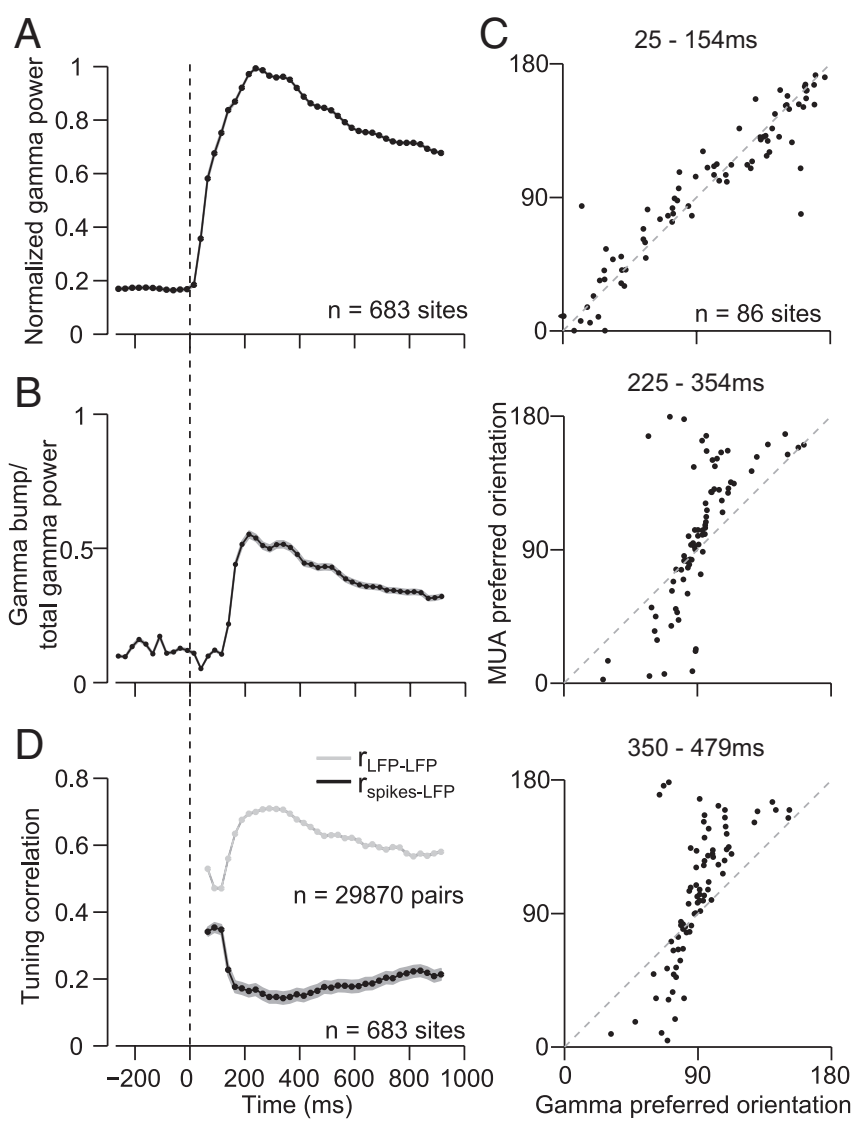

Figure 7. Dynamics of gamma power and its tuning. $\boldsymbol{A}$, Population average of normalized gamma power, as a function of time relative to stimulus onset $(n=683$ sites; error bars are smaller than the line thickness). Power peaks around $250 \mathrm{~ms}$ after stimulus onset. Each point represents the center of one epoch (129 ms window). The dashed vertical line, to facilitate comparisons across plots, indicates stimulus onset at $0 \mathrm{~ms}$. B, Dynamics of the proportion of total power attributable to the gamma bump. $C$, Orientation preference of gamma and MUA for epochs $25-154,225-354$, and $350-479 \mathrm{~ms}$ after stimulus onset ( $n=86$ sites). $\boldsymbol{D}$, Dynamics of $r_{\text {MUA- } \gamma}$ (black; $n=683$ sites) and $r_{\gamma-\gamma}$ (gray trace; $n=29,870$ pairs; error bars are smaller than the line thickness). Negative values indicate time before stimulus onset.

line). The relationship between the tuning of gamma and local MUA is thus dynamic, and these dynamics mirror the relative contribution of the gamma bump to its total power.

In conclusion, there are two components to stimulus-induced increases in gamma power, and the relative weight of these determines the relationship between gamma tuning and that of local spiking activity. Stimuli that induce strong gamma bumps (large, unperturbed gratings) result in similar tuning across sites and a preference that is distinct from that of local MUA. Small gratings, or those masked with noise, induce gamma that arises from a broadband increase in power, resulting in a signal with similar tuning to the local spiking response. Similarly, the dynamic relationship between the tuning of gamma and local spiking activity reveals that, as the contribution of the gamma bump to total gamma power increases, the preference of gamma switches from that of the local MUA to a common orientation across sites.

\section{Spatial coherence of the gamma rhythm}

When gamma power is high and the spectral bump prominent, tuning is similar across sites. This suggests that, under these conditions, the preference of locally measured signals arises from a shared rhythm. To test this directly, we measured the spatial coherence and phase difference of gamma across sites, for different 
A
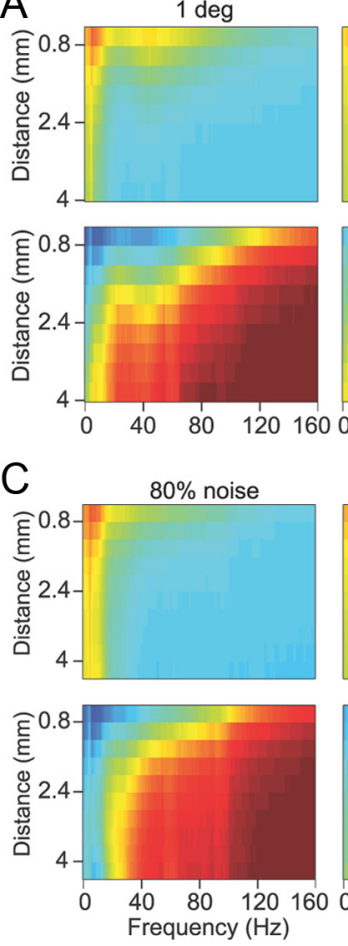

$10 \mathrm{deg}$
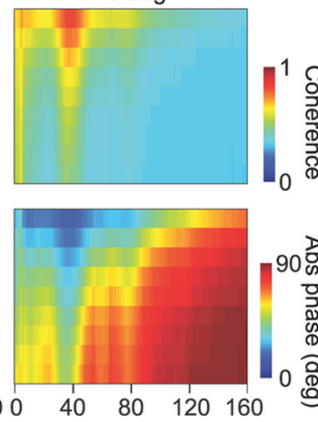
$0 \%$ noise
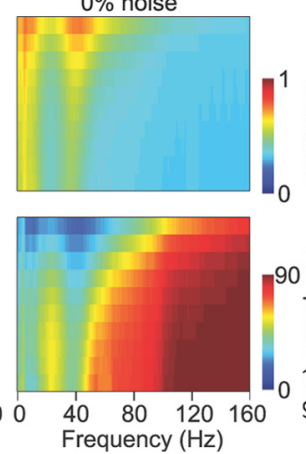

B

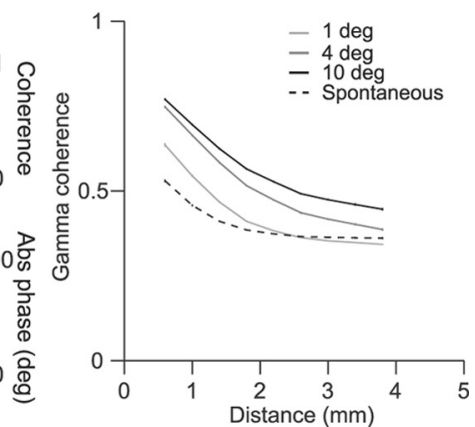

D

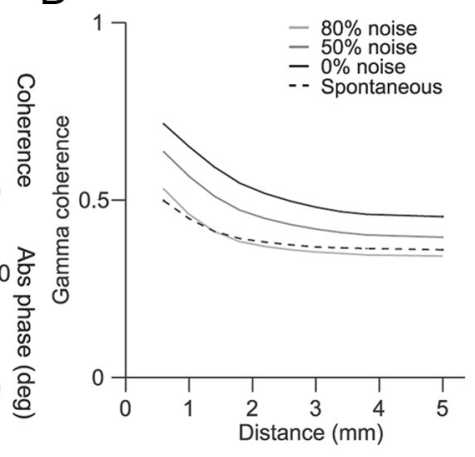

Figure 8. Dependence of gamma coherence and phase alignment on distance. $\boldsymbol{A}$, Coherence (top panels) and absolute phase difference (bottom panels) of the LFP, as a function of the distance between recording sites ( $n=2961$ pairs). Results are shown for signals induced by small gratings ( $1^{\circ}$; left) and large gratings $\left(10^{\circ}\right.$, averaged across stimulus orientations; right). Gamma coherence is weak and has a limited spatial extent when stimuli are small; gamma coherence increases markedly in both magnitude and spatial extent when stimuli are large, especially for the preferred orientation, and gamma activity shows a smaller phase difference across sites. $\boldsymbol{B}$, Coherence of gamma (averaged across orientations) induced by 1,4 , and $10^{\circ}$ gratings as a function of distance between recording sites, compared with that observed in the absence of visual stimulation (spontaneous; dashed line). Error bars, where not visible, are within the line thickness. C, Coherence (top panel) and absolute phase difference (bottom panel) of LFPs induced by $80 \%$ noise masked large grating ( $n=9891$ pairs; left) and unperturbed large gratings (right). $\boldsymbol{D}$, Coherence of gamma induced by 50 and $80 \%$ noise-masked and unperturbed gratings as a function of distance, compared with that observed for spontaneous activity.

stimulus conditions. A shared gamma rhythm would be indicated by higher coherence and smaller phase offsets.

We computed the coherence between signals induced by both large and small stimuli ( $n=2961$ pairs; averaging across different stimulus orientations), using the same set of driven sites. Small gratings $\left(1^{\circ}\right)$ induced a small increase in gamma coherence, relative to spontaneous conditions, with a limited spatial extent of $\sim 2 \mathrm{~mm}$ (Fig. 8 A, left column; B). Large gratings induced a more substantial increase in coherence and signals were also more phase aligned (Fig. $8 \mathrm{~A}$, right column). This enhanced coherence extended across all measured distances (Fig. $8 B$ ) and decayed more slowly for large gratings than small ones (exponential decay with a space constant of $1.6 \mathrm{~mm}$, compared with $1.0 \mathrm{~mm}$ for small gratings). The enhanced coherence for activity induced by large gratings was strongest for the shared, preferred orientation (data not shown).

We also compared coherence for large, unperturbed gratings with those masked with noise. Gamma coherence was weaker and more localized for signals induced by masked gratings (Fig. 8C, left column; $D$ ) ( $n=9891$ pairs). For $80 \%$ masking noise, coherence was nearly indistinguishable from that measured under spontaneous conditions (without stimulus drive), although gamma and spiking responses were selective for such stimuli (as evidenced by the enhanced value of $r_{\text {MUA- } \gamma}$ in Fig. 6).

Our coherence analysis suggests that large gratings induce a single, global gamma rhythm with little phase lag across sites. If

so, gamma activity should be maintained after averaging the signals recorded at different sites on each trial—a signal we term the global LFP. Figure $9 A$ shows singletrial examples of the global LFP induced by large $\left(10^{\circ}\right.$; top) and small $\left(1^{\circ}\right.$; bottom $)$ gratings of the same orientation, averaged across the same sites. The large stimulus induced a prominent gamma rhythm; this was absent from the signal induced by the small stimulus. This result was not due to an undue influence of the signal recorded at a few electrodes, as $Z$-scoring the LFP before averaging yielded essentially identical results. We quantified the power in the global LFP for all frequencies and stimulus sizes we presented ( $n=3 \mathrm{im}$ plants) (Fig. 9B). For small gratings, gamma components of the global LFP had minimal power. For larger stimuli, however, power in the gamma band increased nearly sixfold over the range measured. Other frequency bands did not show this behavior. The global LFP showed strong orientation selectivity for gamma power induced by large but not small stimuli (Fig. 9C). Across implants $(n=3)$, the selectivity of the gamma component of the global LFP was $0.32 \pm 0.08$, comparable with the selectivity at each individual site $(0.37 \pm 0.01)$, and its preferred orientation closely matched the mean preference of signals measured at individual sites (mean offset of $1.7 \pm 0.7^{\circ} ; n=8$ implants).

Together, these results show that the gamma measured at each electrode reflects a spatially extensive rhythm, when activity is induced by large but not small gratings. The power in this signal is stronger for some orientations than others, giving rise to a common preference across sites. A potential explanation for the extensive coherence of gamma induced by large stimuli is that it reflects volume conduction, which could in principle explain its similar tuning across sites as well (i.e., if the signal measured by each electrode reflects a spatial average of distantly generated gamma signals). Several pieces of evidence argue against this. First, recent estimates of volume conduction suggest it is limited to $250 \mu \mathrm{m}$ (Xing et al., 2009), 20-fold smaller than the extent of spatially coherent gamma that we observe. Consistent with this, annular gratings induce no gamma power at sites where spiking activity is not elevated (Gieselmann and Thiele, 2008) (X. Jia and A. Kohn, unpublished observations), showing that gamma from distant sites does not passively propagate over large distances. Second, because high-amplitude signals should conduct more effectively (i.e., remain measurable despite the attenuation associated with passive propagation through the extracellular space), one would expect low frequencies to be more coherent than gamma frequencies, as their power is substantially higher (Fig. $1 B$ ). However, when induced by large gratings, gamma was slightly more coherent than low frequencies (Fig. $8 \mathrm{~A}$ ) (mean of $0.599 \pm 0.002$ for gamma vs $0.583 \pm 0.002$ for frequencies $<10 \mathrm{~Hz}$ ), although gamma power was 26 -fold lower. Note also that the change in the preference and coherence of gamma with stimulus conditions 

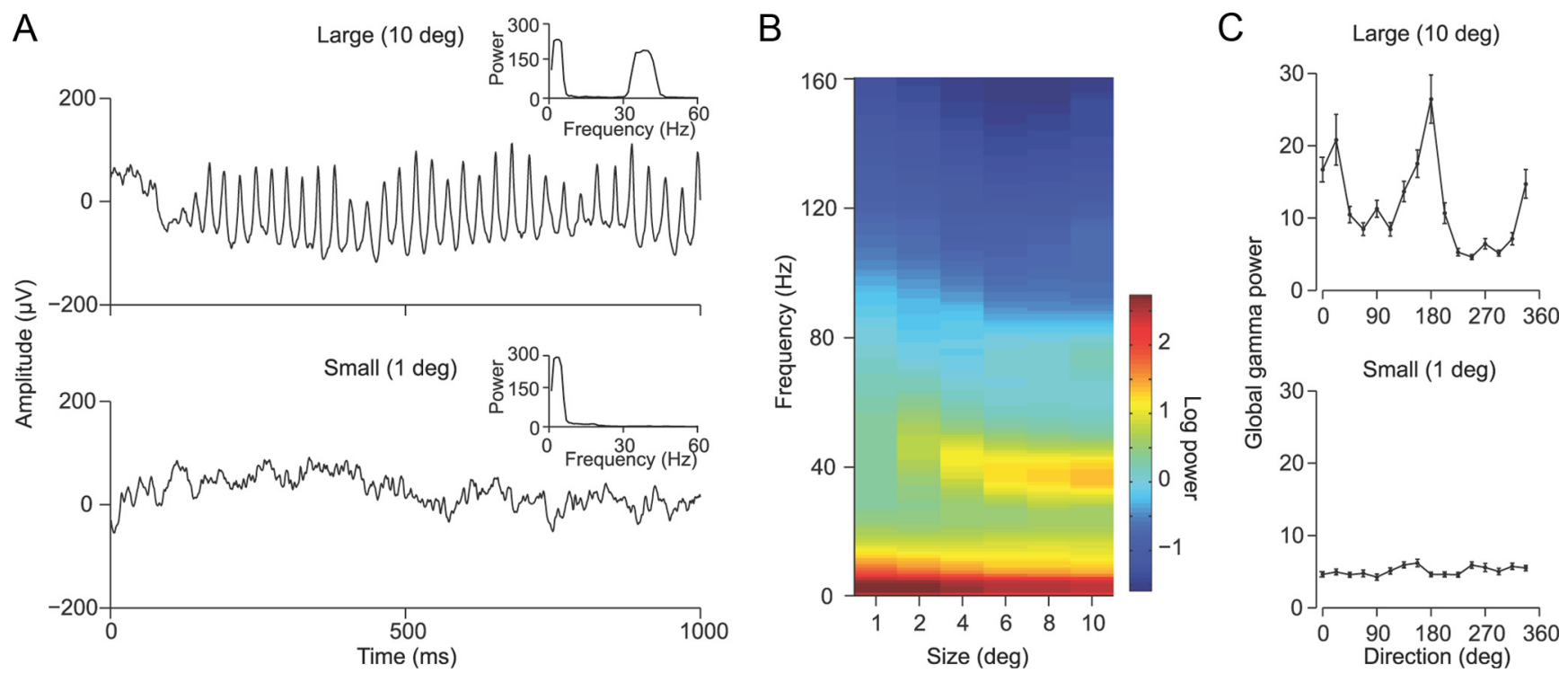

Figure 9. The global LFP.A, Single trial examples of the global LFP averaged across 31 sites of one implant, for activity induced by a large (top) and small (bottom) stimulus of the same orientation. The insets show the power spectrum of the signal. $\boldsymbol{B}$, Average of the global LFP power spectra for different sizes ( $n=3$ implants). Value indicated is the average across orientations. $\boldsymbol{C}$, Orientation tuning of the gamma component of the global LFP, for $10^{\circ}$ (top) and $1^{\circ}$ (bottom) stimuli of an array implant.

involved roughly twofold changes in power (Figs. $4 A, 6 A)$. It seems unlikely that this would result in a signal whose tuning was different at sites separated by $400 \mu \mathrm{m}$, in one case, but shared up to $9 \mathrm{~mm}$ in another. Third, when a strong gamma rhythm is induced, spike-spike coherence in gamma frequencies is elevated across the array (Jia et al., 2011). Since volume-conducted fields have little influence on membrane potential-compared with those locally generated-the enhanced coordination of neuronal activity argues strongly against an important contribution of volume conduction (Bauer et al., 2007). Fourth, if the gamma rhythm induced by large gratings involved simple volume conduction of distant signals, one would expect little or no selectivity, because each site would represent the average of locally generated signals with different preferences. In fact, tuning was more selective for gamma induced by large gratings than small ones (as described above).

\section{An adaptable bias underlies the preference of the global gamma rhythm}

We have shown that large stimuli induce a spatially extensive gamma rhythm that is both well tuned and has a common preference across millimeters of cortex. We wondered why some orientations would induce a stronger rhythm than others, over such a large region. One possibility is that this preferred orientation reflects or magnifies a small bias in the neuronal representation of orientation. An obvious source for this bias would be the purported systematic overrepresentation of cardinal orientations in primary visual cortex ( $\mathrm{Li}$ et al., 2003). However, we observed a shared preference for noncardinal orientations in a number of implants, such as those in Figure 2, $C$ and $D$. Alternatively, the shared preference could arise from an inhomogeneous representation within a more limited region, such as the bias seen in fMRI voxels that are orientation-tuned despite reflecting activity averaged over several (or even many) cubic millimeters (Kamitani and Tong, 2005; Haynes and Rees, 2006).

We attempted to detect this potential representational bias with our neuronal recordings by comparing the preference of the gamma induced by large gratings to the most common preference of the spiking activity detected by our array. Figure $10 \mathrm{~A}$ shows the range of MUA preferences from one array $\left(\sigma^{2}=0.88\right.$; $n=89$ sites, arranged according to their position on the array), measured with large gratings. Figure $10 \mathrm{~B}$ shows the orientation preference of gamma from the same array, with its characteristic narrow distribution of preferences $\left(\sigma^{2}=0.26\right)$. In this implant, there is no obvious relationship between the preference distribution of gamma and that of the recorded spiking responses: the preferred orientation of gamma is near $0^{\circ}$, but there is no bias for this orientation in the spiking responses. We quantified this relationship by computing the correlation between the populationaveraged tuning of MUA and gamma, after normalizing the data for each site by the maximal response. For the example implant, this correlation was 0.28 (Fig. 10C). Across implants the mean correlation was $0.27 \pm 0.24(n=8)$, not significantly different from zero $(p=0.72)$. We were thus unable to detect a bias in the spiking representation of orientation that could underlie the shared preference of gamma. However, this failure is perhaps not surprising given the limited sample of recording sites provided by the array.

Because we could not observe a relationship between the preferences of the sampled spiking activity and the shared preference of gamma, we used an alternative strategy of manipulating the representation of orientation with adaptation. Adaptation is well known to reduce the responsivity of cortical neurons whose preferences match the adapter and to have little effect on cells with offset preferences (Kohn, 2007). If gamma magnifies a presumed bias in the neuronal representation of orientation, adaptationinduced changes in neuronal response should have a strong consequence for gamma tuning.

We measured tuning with our standard grating stimuli, before and after adapting with a single orientation for $40 \mathrm{~s}$. To maintain the effects of adaptation, we used brief ( $5 \mathrm{~s}$ ) "top-up" adaptation between test stimuli (Fig. 11A). An illustration of how adaptation affects the orientation preference of gamma is shown in Figure $11 B$ for a recording in which the adapter (indicated by the arrow) was well aligned with the most commonly preferred orientation. Adaptation caused a striking shift in the preference of gamma to 


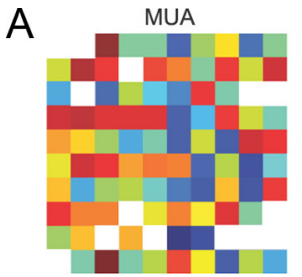

B

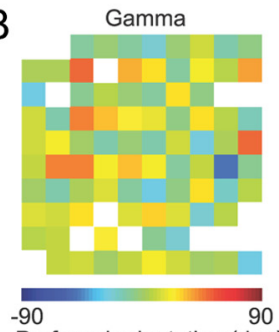

Preferred orientation (deg)

C

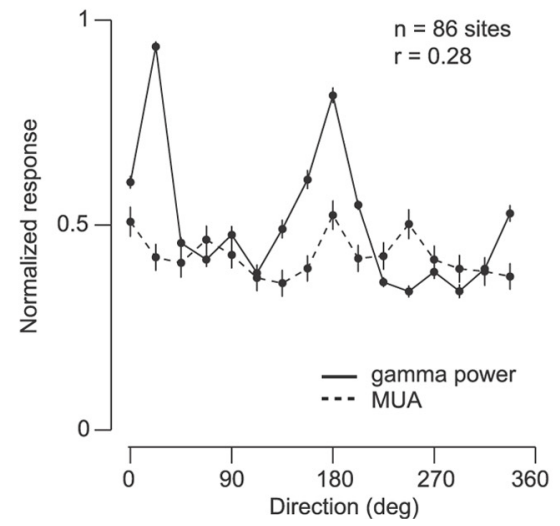

Figure 10. Relationship between neuronal preferences sampled by the array and the preference of gamma. $A$, MUA orientation preferences plotted according to electrode positions on the array (left) and corresponding distribution (right). $\boldsymbol{B}$, Gamma preferences from the same array as in $\boldsymbol{A}$ and their distribution ( $n=86$ sites). $\boldsymbol{C}$, Population tuning curves of MUA (dashed) and gamma (solid) calculated by averaging normalized tuning curves of all sites from the same array. The two tuning curves are only weakly related, with a correlation of 0.28
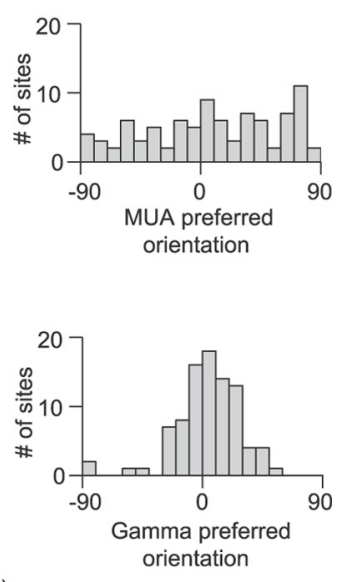

ing curves, computed by averaging data from sites with similar preadaptation preferences (bins of $22.5^{\circ}$ ) after normalizing the responses at each site by its maximum. Adaptation caused a dramatic shift of preference to the orthogonal orientation when the adapter was well matched to the initial preference (Fig. $11 D$, top) $\left(n=127\right.$ sites; shift of $\left.65.4^{\circ}\right)$. This was due to a strong suppression of the gamma induced by stimuli similar to the adapter, and a facilitation of the signal induced by orthogonal orientations. In recordings for which the preadaptation gamma preference was orthogonal to the adapter, these effects led to little change in preference but a clearer shared tuning (Fig. $11 \mathrm{D}$, bottom) $\left(n=138\right.$ sites; shift of $\left.4.0^{\circ}\right)$. These results suggest that the global gamma rhythm is extremely sensitive to adaptation-induced changes in neural representation, consistent with its preference magnifying a weak bias in that representation.

We tested our explanation for the shared preference of gamma in an additional, independent manner: by measuring tuning for grating spatial and temporal frequency. Whereas the representation of orientation would be expected to be at most weakly biased, neuronal preferences for spatial and temporal frequency are well known to be nonuniformly distributed (Foster et al., 1985; Hawken et al., 1996; O'Keefe et al., 1998). We reasoned that, if a biased representation underlies the preference of gamma, its tuning for spatial and temporal frequency should be similar both across sites and implants.

We measured tuning using large gratings drifting at a fixed drift rate $(6.25$ cycles/s) and with spatial frequencies ranging from 0.1 to $8.6 \mathrm{cpd}$, or with a fixed spatial frequency ( $1 \mathrm{cpd}$ ) and a range of drift rates $(0.3-25$ cycles/s). Sites for which the minimal response was not at least $50 \%$ smaller than the peak response were deemed untuned (Foster et al., 1985) (6.3\% of 567 MUA sites for spatial frequency and $9.6 \%$ of 732 MUA sites for temporal fre-
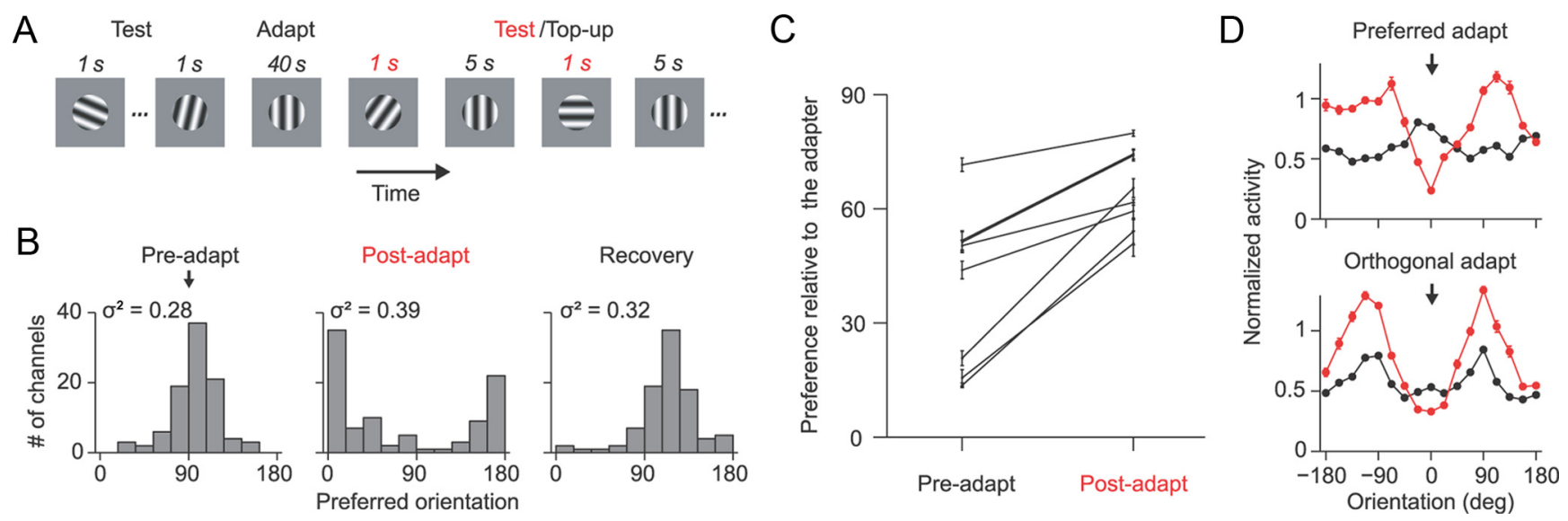

Figure 11. Effect of adaptation on the tuning of gamma. $\boldsymbol{A}$, Illustration of the adaptation protocol. $\boldsymbol{B}$, Distributions (from a single implant) of orientation preference for preadaptation, postadaptation, and recovery periods for gamma (top) and MUA (bottom). The circular variance is indicated at the top left of each histogram. The arrow indicates the orientation of the adapter. $\boldsymbol{C}$, Quantification of mean orientation preference of gamma, relative to the adapter, before and after adaptation ( $n=8$ implants). $\boldsymbol{D}$, Population-averaged orientation tuning curves before (black) and after (red) adaptation for gamma. All tuning curves are aligned so that 0 represents the adapting orientation (indicated by arrow). The top panels represent cases in which the tuning preference was within $22.5^{\circ}$ of the adapter ( $n=127$ sites). The bottom panels are cases in which the preference was orthogonal to the adapter ( $n=138$ sites). 

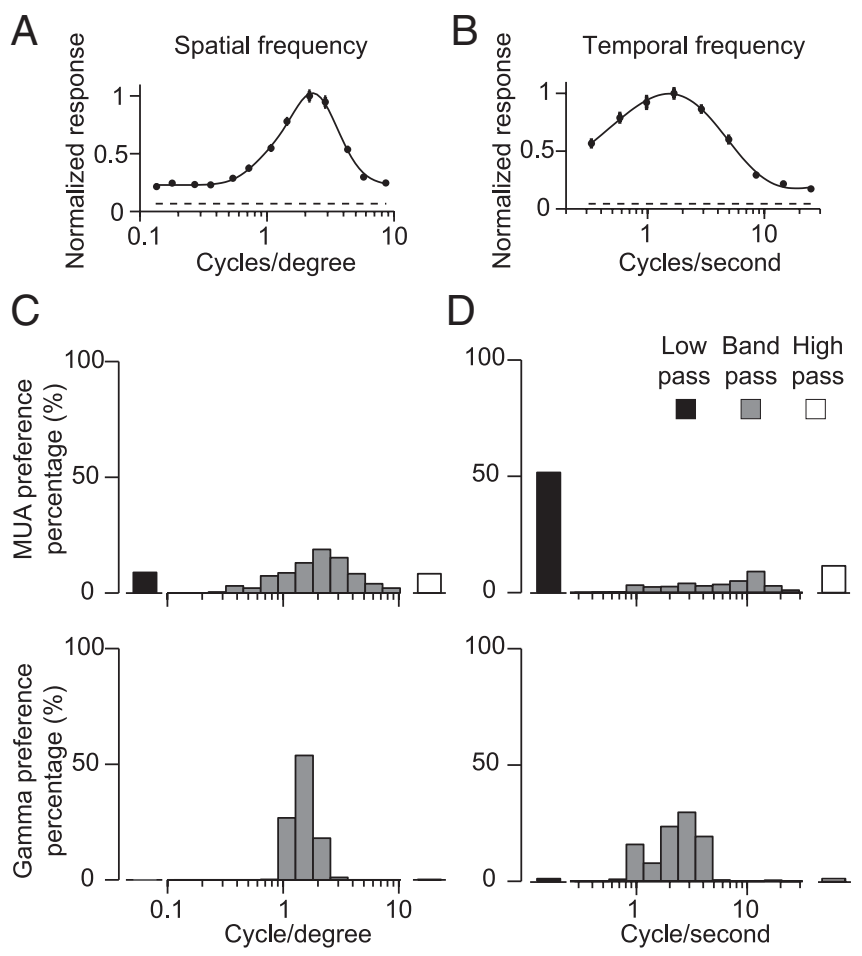

Figure 12. MUA and gamma tuning for stimulus spatial and temporal frequency. $\boldsymbol{A}, \mathrm{An}$ example of gamma tuning for spatial frequency for a single site. $\boldsymbol{B}$, Same as $\boldsymbol{A}$ for temporal frequency tuning. $C$, Distribution of spatial frequency preferences across all implants ( 567 sites from 7 implants), for tuned sites of MUA (top) and gamma (bottom). D, Same as C for temporal frequency preferences (732 sites from 9 implants).

quency). At selective sites, MUA often had high-pass or low-pass tuning (Fig. 12C,D) (7.9 and 9.1\%, respectively, for spatial frequency; and 10.3 and $36.0 \%$ for temporal frequency), defined as a response at the lowest or highest measured frequency that was $>75 \%$ of the peak response (Levitt et al., 1994; Movshon et al., 2005). At the remaining bandpass sites, we estimated the preference based on the fit of a difference-of-Gaussians function to the data. MUA had a wide range of spatial and temporal frequency preferences (Fig. 12C,D).

Unlike the tuning of MUA, gamma power had bandpass tuning for both stimulus parameters at almost all sites (Fig. 12 A,B) (99.8\% of sites for spatial frequency tuning and $97.8 \%$ for temporal frequency). There were no untuned gamma sites. Remarkably, gamma preferences for spatial and temporal frequency were similar across sites and implants (Fig. 12C,D). For instance, across 567 sites recorded in seven implants, $86 \%$ of sites preferred a spatial frequency between 1 and $3 \mathrm{cpd}$; for MUA, these preferences were most common but they accounted for only $29 \%$ of sites. The similarity of spatial and temporal frequency tuning of gamma, across sites and implants, is consistent with its preference arising from the biased representation for these stimulus features.

\section{Discussion}

To determine the spatial extent of gamma and its relationship to spiking activity, we recorded LFPs and MUA simultaneously in the upper layers of macaque V1. We found that gamma could have similar tuning to local spiking activity, when it reflected a broadband increase in power. Under other conditions, gamma could form a coherent, spatially extensive rhythm with similar tuning across millimeters of cortex. This latter behavior was evident for stimuli that induced a distinct spectral bump, a signal that grew slowly after stimulus onset and that could be disrupted by reducing stimulus size, or using masking noise. Our results suggest two distinct components to gamma power, with very different relationships to neuronal activity. The relative weight of these two components is stimulus dependent, resulting in a flexible relationship between the tuning of gamma power and local spiking activity.

This flexible relationship offers an explanation for previous disparate findings. Some previous comparisons of the tuning of gamma and local MUA have found matched preferences, but others have not. This has led to estimates that gamma reflects activity within $250 \mu \mathrm{m}$ up to $3 \mathrm{~mm}$ of the electrode (Gray and Singer, 1989; Kreiman et al., 2006; Liu and Newsome, 2006; Berens et al., 2008; Gieselmann and Thiele, 2008). Our findings show stimulus conditions under which each of these descriptions is accurate. Similarly, studies of the relationship between gamma and the BOLD fMRI signal have found both strong correlation [Kayser et al., 2004; Goense and Logothetis, 2008 (who both used prolonged presentations of large gratings)] and a weak one [Maier et al., 2008 (who used briefly flashed noise stimuli)]. These differences could be explained by the two components of gamma we observe, assuming the global gamma rhythm is more directly related to the macroscopic measurement afforded by BOLD. More generally, our results emphasize that one must carefully consider stimulus properties - and the relative weight of broadband increases in power to the spectral bump-in understanding and interpreting the behavior of gamma.

When induced by large gratings, gamma is coherent over millimeters of cortex, consistent with previous studies (Juergens et al., 1999; Frien and Eckhorn, 2000; Leopold et al., 2003). Despite this spatial extent, it remains remarkably well tuned. Our observations thus reconcile previous work emphasizing the extent of gamma coherence with seemingly inconsistent claims that gamma is well tuned. Importantly, our results show that inferring the spatial extent of gamma rhythms from their selectivity is problematic: a well tuned signal does not mean a local one. Only by measuring tuning and coherence across sites - a novel feature of our study - can one accurately determine the extent and functional specificity of gamma rhythms.

Our findings do not contradict recent reports that the evoked LFP reflects neural activity within $250 \mu \mathrm{m}$ of recording site (Katzner et al., 2009) and that the passive propagation of extracellular fields has a similarly limited spatial extent (Xing et al., 2009). We focused on an induced signal whose extent is influenced by passive propagation but ultimately determined by the circuits generating it. Katzner et al. (2009) did compare the orientation tuning of the induced and evoked LFP and concluded they were similar. However, activity was driven by brief stimuli (32 $\mathrm{ms}$ ) that do not induce a strong gamma rhythm (Fig. 7) (Kruse and Eckhorn, 1996). To be sure that our different conclusions concerning induced gamma were not due to other factors, we recorded responses to similarly brief $(40 \mathrm{~ms})$ presentations and confirmed that the induced gamma power is weak, contains little evidence of a bump, and that both this signal and the evoked response are similarly tuned to local MUA (data not shown), entirely consistent with Katzner et al. (2009).

A previous study by Berens et al. (2008) conducted in awake macaque V1 using large gratings, reported findings similar to a subset of ours. Namely, they found that the orientation preference of gamma was similar across nearby sites (separated by 1 $\mathrm{mm}$ or less) and inferred that gamma reflects activity within 500 $1000 \mu \mathrm{m}$ of the electrode tip, due to a combination of extensive circuits generating the rhythm and the volume conduction of 
those signals. Our interpretation differs from theirs in part because we show that gamma power consists of two components. Shared tuning across sites is not a fixed property of gamma; it occurs only when a prominent bump is induced. Furthermore, our data suggest that, when tuning is similar across sites, this is not due to simple volume conduction of nearby signals (for reasons described in Results), but rather because of the formation of a spatially extensive, coherent rhythm. Our different interpretation arises because we considered a wider range of stimulus manipulations (size, noise masking, dynamics, and spatial and temporal frequency), sampled across a $\sim 10$-fold higher range of distances, and because we analyzed both coherence and tuning across sites.

Berens et al. (2008) also reported that the selectivity of gamma is positively correlated with its similarity to local spiking activity. They propose that this relationship reflects the spatially extensive ensemble contributing to gamma and whether the recording site is situated in an iso-orientation domain (similar preference to MUA and high selectivity) or at a pinwheel center. We observe a similar relationship between selectivity and the match to local MUA (Jia and Kohn, unpublished observations), even when gamma was induced by large gratings and extends over many pinwheels. In this case, the correlation may arise because when gamma preference is matched to local spiking activity, the gamma bump and its broadband component will have a similar preference, resulting in higher selectivity; at sites where gamma is different from local spiking activity, these two components will have different preferences, resulting in weaker selectivity.

\section{Mechanisms}

The mechanisms of gamma generation have been studied extensively, both in the hippocampus and neocortex. GABAergic interneurons have been shown to play a critical role in generating gamma (Whittington et al., 1995, 2011; Traub et al., 1996a; Hasenstaub et al., 2005; Bartos et al., 2007; Atallah and Scanziani, 2009; Cardin et al., 2009), and this is perhaps enhanced by interactions with excitatory neurons (Buzsaki, 2006; Tiesinga and Sejnowski, 2009). In cortex, "chattering cells" may also contribute (Gray and McCormick, 1996; Cunningham et al., 2004).

These mechanisms do not appear to contribute strongly to the gamma induced by small stimuli and large, masked gratings, or at response onset. This is because gamma in these cases reflects a broadband increase in power, rather than a mechanism specific to gamma frequencies (Ray and Maunsell, 2010). Instead, the broadband increase in power likely arises from a general elevation of synaptic and spiking activity in local circuits because this signal behaves similarly to local MUA (e.g., similar orientation tuning and suppression by large gratings). This component of gamma may also include direct spectral contamination of the LFP by spiking activity, although previous work suggests this is limited to frequency components $>50 \mathrm{~Hz}$ (David et al., 2010; Zanos et al., 2011).

The traditional inhibitory (or excitatory-inhibitory) mechanisms of gamma generation presumably do underlie the spectral bump we observe for activity induced by large stimuli. However, the global nature of this rhythm suggests additional spatially extensive mechanisms that coordinate or drive this inhibitory network. One possibility is that this involves feedback connections, which extend over long distances and are thought to contribute to the surround suppression recruited by large stimuli (Bair et al., 2003; Angelucci and Bressloff, 2006). Alternatively, the global gamma rhythm may be an emergent rhythm that involves the coordination of local generators through mechanisms such as long-range lateral connections or gap-junction coupling among inhibitory neurons (Traub et al., 1996b; Gibson et al., 1999; Buzsaki, 2006; Tiesinga and Sejnowski, 2009). Finally, the global gamma rhythm may arise from an altered balance between excitatory and inhibitory activity in cortex, because inhibitory cells may be only weakly surround suppressed (Brunel and Wang, 2003; Haider et al., 2010).

Whichever mechanisms contribute, they must be more effective for some stimuli than others, a property we suggest may be due to biased representations for these features (e.g., stronger feedback or an enhanced local representation). An intriguing possibility is that this bias involves a recently described overrepresentation of orientations corresponding to the radial position of the spatial receptive fields [a radial bias (Freeman et al., 2011)]. The sites we recorded represented similar positions in the visual field across animals, so one might expect to observe similar preferences. However, the gratings we used were positioned slightly differently in each animal, and given the proximity of our recordings to the fovea, these small differences could alter the net bias in the recruited population. These explanations for our findings are, of course, speculative, and additional work is needed to address the mechanisms underlying the novel properties of gamma we report.

\section{Functional role of gamma rhythms}

Through its suggested influence on spike timing, gamma has been proposed to provide a temporal window for communication (Fries, 2009), encode the amplitude of stimulus drive in response phase (Fries et al., 2007), bind distributed representations (Gray, 1999), or dynamically route information (Pesaran et al., 2002; Colgin et al., 2009; Fries, 2009). The spatially extensive, coherent gamma rhythm we observe seems better suited for an integrative function than one that requires targeting specific subsets of neurons. For instance, the gamma induced by a large vertical grating is similar at sites where neurons prefer vertical or horizontal structure. Thus, it could not be expected to emphasize or group specific subset of neurons. Only when gamma power is limited (reflecting a broadband increase in power), is it spatially and functionally selective. However, precisely because it is weak, it is unlikely to have a strong influence on spike timing under these conditions (Sohal et al., 2009; Okun et al., 2010).

As an integrative signal, the global gamma rhythm could, in principle, function as an internal reference. For instance, it seems well suited to modulate spike timing in a large region of cortex. However, this rhythm is much stronger for some stimuli than others, in a patch of cortex representing several degrees of visual field. Unless perceptual performance is similarly biased, this would suggest a limited functional role, at least for stimulusinduced gamma activity in V1. Under this interpretation, the global gamma rhythm may simply be a resonant frequency arising from the interaction between excitation and inhibition (Burns et al., 2010; Ray and Maunsell, 2010), albeit one that can reflect a much more extensive ensemble than previously considered.

\section{References}

Angelucci A, Bressloff PC (2006) Contribution of feedforward, lateral and feedback connections to the classical receptive field center and extraclassical receptive field surround of primate V1 neurons. Prog Brain Res 154:93-120.

Atallah BV, Scanziani M (2009) Instantaneous modulation of gamma oscillation frequency by balancing excitation with inhibition. Neuron 62:566-577.

Bair W, Cavanaugh JR, Movshon JA (2003) Time course and time-distance relationships for surround suppression in macaque V1 neurons. J Neurosci 23:7690-7701 
Bartos M, Vida I, Jonas P (2007) Synaptic mechanisms of synchronized gamma oscillations in inhibitory interneuron networks. Nat Rev Neurosci 8:45-56.

Bauer EP, Paz R, Paré D (2007) Gamma oscillations coordinate amygdalorhinal interactions during learning. J Neurosci 27:9369-9379.

Bauer R, Brosch M, Eckhorn R (1995) Different rules of spatial summation from beyond the receptive field for spike rates and oscillation amplitudes in cat visual cortex. Brain Res 669:291-297.

Berens P, Keliris GA, Ecker AS, Logothetis NK, Tolias AS (2008) Feature selectivity of the gamma-band of the local field potential in primate primary visual cortex. Front Neurosci 2:199-207.

Brosch M, Budinger E, Scheich H (2002) Stimulus-related gamma oscillations in primate auditory cortex. J Neurophysiol 87:2715-2725.

Brunel N, Wang XJ (2003) What determines the frequency of fast network oscillations with irregular neural discharges? I. Synaptic dynamics and excitation-inhibition balance. J Neurophysiol 90:415-430.

Burns SP, Xing D, Shelley MJ, Shapley RM (2010) Searching for autocoherence in the cortical network with a time-frequency analysis of the local field potential. J Neurosci 30:4033-4047.

Buzsaki G (2006) Rhythms of the brain. New York: Oxford UP.

Cardin JA, Carlén M, Meletis K, Knoblich U, Zhang F, Deisseroth K, Tsai LH, and Moore CI (2009) Driving fast-spiking cells induces gamma rhythm and controls sensory responses. Nature 459:663-667.

Colgin LL, Denninger T, Fyhn M, Hafting T, Bonnevie T, Jensen O, Moser $\mathrm{MB}$, Moser EI (2009) Frequency of gamma oscillations routes flow of information in the hippocampus. Nature 462:353-357.

Cunningham MO, Whittington MA, Bibbig A, Roopun A, LeBeau FE, Vogt A, Monyer H, Buhl EH, Traub RD (2004) A role for fast rhythmic bursting neurons in cortical gamma oscillations in vitro. Proc Natl Acad Sci U S A 101:7152-7157.

David SV, Malaval N, Shamma SA (2010) Decoupling action potential bias from cortical local field potentials. Comput Intell Neurosci 393019.

Foster KH, Gaska JP, Nagler M, Pollen DA (1985) Spatial and temporal frequency selectivity of neurones in visual cortical areas V1 and V2 of the macaque monkey. J Physiol 365:331-363.

Freeman J, Brouwer GJ, Heeger DJ, Merriam EP (2011) Orientation decoding depends on maps, not columns. J Neurosci 31:4792-4804.

Frien A, Eckhorn R (2000) Functional coupling shows stronger stimulus dependency for fast oscillations than for low-frequency components in striate cortex of awake monkey. Eur J Neurosci 12:1466-1478.

Frien A, Eckhorn R, Bauer R, Woelbern T, Gabriel A (2000) Fast oscillations display sharper orientation tuning than slower components of the same recordings in striate cortex of the awake monkey. Eur J Neurosci 12:1453-1465.

Fries P (2009) Neuronal gamma-band synchronization as a fundamental process in cortical computation. Annu Rev Neurosci 32:209-224.

Fries P, Nikolić D, Singer W (2007) The gamma cycle. Trends Neurosci 30:309-316.

Fries P, Womelsdorf T, Oostenveld R, Desimone R (2008) The effects of visual stimulation and selective visual attention on rhythmic neuronal synchronization in macaque area V4. J Neurosci 28:4823-4835.

Gail A, Brinksmeyer HJ, Eckhorn R (2004) Perception-related modulations of local field potential power and coherence in primary visual cortex of awake monkey during binocular rivalry. Cereb Cortex 14:300-313.

Gibson JR, Beierlein M, Connors BW (1999) Two networks of electrically coupled inhibitory neurons in neocortex. Nature 402:75-79.

Gieselmann MA, Thiele A (2008) Comparison of spatial integration and surround suppression characteristics in spiking activity and the local field potential in macaque V1. Eur J Neurosci 28:447-459.

Goense JB, Logothetis NK (2008) Neurophysiology of the BOLD fMRI signal in awake monkeys. Curr Biol 18:631-640.

Gray CM (1999) The temporal correlation hypothesis of visual feature integration: still alive and well. Neuron 24:31-47, 111-125.

Gray CM, McCormick DA (1996) Chattering cells: superficial pyramidal neurons contributing to the generation of synchronous oscillations in the visual cortex. Science 274:109-113.

Gray CM, Singer W (1989) Stimulus-specific neuronal oscillations in orientation columns of cat visual cortex. Proc Natl Acad Sci USA 86:1698-1702.

Haider B, Krause MR, Duque A, Yu Y, Touryan J, Mazer JA, McCormick DA (2010) Synaptic and network mechanisms of sparse and reliable visual cortical activity during nonclassical receptive field stimulation. Neuron 65:107-121

Hasenstaub A, Shu Y, Haider B, Kraushaar U, Duque A, McCormick DA (2005) Inhibitory postsynaptic potentials carry synchronized frequency information in active cortical networks. Neuron 47:423-435.

Hawken MJ, Shapley RM, Grosof DH (1996) Temporal-frequency selectivity in monkey visual cortex. Vis Neurosci 13:477-492.

Haynes JD, Rees G (2006) Decoding mental states from brain activity in humans. Nat Rev Neurosci 7:523-534.

Henrie JA, Shapley R (2005) LFP power spectra in V1 cortex: the graded effect of stimulus contrast. J Neurophysiol 94:479-490.

Hopfield JJ (2004) Encoding for computation: recognizing brief dynamical patterns by exploiting effects of weak rhythms on action-potential timing. Proc Natl Acad Sci U S A 101:6255-6260.

Jia X, Zandvakili A, Kohn A (2011) Gamma rhythms, neuronal synchrony, and corticocortical communication in early visual cortex. Paper presented at COSYNE 2011, Salt Lake City, February.

Juergens E, Guettler A, Eckhorn R (1999) Visual stimulation elicits locked and induced gamma oscillations in monkey intracortical- and EEGpotentials, but not in human EEG. Exp Brain Res 129:247-259.

Kamitani Y, Tong F (2005) Decoding the visual and subjective contents of the human brain. Nat Neurosci 8:679-685.

Katzner S, Nauhaus I, Benucci A, Bonin V, Ringach DL, Carandini M (2009) Local origin of field potentials in visual cortex. Neuron 61:35-41.

Kayser C, Kim M, Ugurbil K, Kim DS, König P (2004) A comparison of hemodynamic and neural responses in cat visual cortex using complex stimuli. Cereb Cortex 14:881-891.

Kohn A (2007) Visual adaptation: physiology, mechanisms, and functional benefits. J Neurophysiol 97:3155-3164.

Kreiman G, Hung CP, Kraskov A, Quiroga RQ, Poggio T, DiCarlo JJ (2006) Object selectivity of local field potentials and spikes in the macaque inferior temporal cortex. Neuron 49:433-445.

Kruse W, Eckhorn R (1996) Inhibition of sustained gamma oscillations $(35-80 \mathrm{~Hz})$ by fast transient responses in cat visual cortex. Proc Natl Acad Sci U S A 93:6112-6117.

Leopold DA, Murayama Y, Logothetis NK (2003) Very slow activity fluctuations in monkey visual cortex: implications for functional brain imaging. Cereb Cortex 13:422-433.

Leventhal AG, Thompson KG, Liu D, Zhou Y, Ault SJ (1995) Concomitant sensitivity to orientation, direction, and color of cells in layers 2, 3, and 4 of monkey striate cortex. J Neurosci 15:1808-1818.

Levitt JB, Kiper DC, Movshon JA (1994) Receptive fields and functional architecture of macaque V2. J Neurophysiol 71:2517-2542.

Li B, Peterson MR, Freeman RD (2003) Oblique effect: a neural basis in the visual cortex. J Neurophysiol 90:204-217.

Lima B, Singer W, Chen NH, Neuenschwander S (2010) Synchronization dynamics in response to plaid stimuli in monkey V1. Cereb Cortex 20:1556-1573.

Liu J, Newsome WT (2006) Local field potential in cortical area MT: stimulus tuning and behavioral correlations. J Neurosci 26:7779-7790.

Maier A, Wilke M, Aura C, Zhu C, Ye FQ, Leopold DA (2008) Divergence of $\mathrm{fMRI}$ and neural signals in $\mathrm{V} 1$ during perceptual suppression in the awake monkey. Nat Neurosci 11:1193-1200.

Mitra PP, Pesaran B (1999) Analysis of dynamic brain imaging data. Biophys J 76:691-708.

Mitzdorf U (1985) Current source-density method and application in cat cerebral cortex: investigation of evoked potentials and EEG phenomena. Physiol Rev 65:37-100.

Movshon JA, Kiorpes L, Hawken MJ, Cavanaugh JR (2005) Functional maturation of the macaque's lateral geniculate nucleus. J Neurosci 25:2712-2722.

Murthy VN, Fetz EE (1992) Coherent 25- to 35-Hz oscillations in the sensorimotor cortex of awake behaving monkeys. Proc Natl Acad Sci U S A 89:5670-5674.

O’Keefe LP, Levitt JB, Kiper DC, Shapley RM, Movshon JA (1998) Functional organization of owl monkey lateral geniculate nucleus and visual cortex. J Neurophysiol 80:594-609.

Okun M, Naim A, Lampl I (2010) The subthreshold relation between cortical local field potential and neuronal firing unveiled by intracellular recordings in awake rats. J Neurosci 30:4440-4448.

Pesaran B, Pezaris JS, Sahani M, Mitra PP, Andersen RA (2002) Temporal 
structure in neuronal activity during working memory in macaque parietal cortex. Nat Neurosci 5:805-811.

Popescu AT, Popa D, Paré D (2009) Coherent gamma oscillations couple the amygdala and striatum during learning. Nat Neurosci 12:801-807.

Ray S, Maunsell JH (2010) Differences in gamma frequencies across visual cortex restrict their possible use in computation. Neuron 67:885-896.

Schoffelen JM, Oostenveld R, Fries P (2005) Neuronal coherence as a mechanism of effective corticospinal interaction. Science 308:111-113.

Siegel M, König P (2003) A functional gamma-band defined by stimulusdependent synchronization in area 18 of awake behaving cats. J Neurosci 23:4251-4260.

Smith MA, Kohn A (2008) Spatial and temporal scales of neuronal correlation in primary visual cortex. J Neurosci 28:12591-12603.

Smith MA, Kelly RC, Lee TS (2007) Dynamics of response to perceptual pop-out stimuli in macaque V1. J Neurophysiol 98:3436-3449.

Sohal VS, Zhang F, Yizhar O, Deisseroth K (2009) Parvalbumin neurons and gamma rhythms enhance cortical circuit performance. Nature 459:698-702.

Tallon-Baudry C (2003) Oscillatory synchrony and human visual cognition. J Physiol Paris 97:355-363.

Tiesinga P, Sejnowski TJ (2009) Cortical enlightenment: are attentional gamma oscillations driven by ING or PING? Neuron 63:727-732.

Traub RD, Whittington MA, Colling SB, Buzsáki G, Jefferys JG (1996a)
Analysis of gamma rhythms in the rat hippocampus in vitro and in vivo. J Physiol 493:471-484.

Traub RD, Whittington MA, Stanford IM, Jefferys JG (1996b) A mechanism for generation of long-range synchronous fast oscillations in the cortex. Nature 383:621-624.

Whittington MA, Traub RD, Jefferys JG (1995) Synchronized oscillations in interneuron networks driven by metabotropic glutamate receptor activation. Nature 373:612-615.

Whittington MA, Cunningham MO, LeBeau FE, Racca C, Traub RD (2011) Multiple origins of the cortical gamma rhythm. Dev Neurobiol 71:92-106.

Wilke M, Logothetis NK, Leopold DA (2006) Local field potential reflects perceptual suppression in monkey visual cortex. Proc Natl Acad Sci U S A 103:17507-17512.

Womelsdorf T, Fries P, Mitra PP, Desimone R (2006) Gamma-band synchronization in visual cortex predicts speed of change detection. Nature 439:733-736.

Xing D, Yeh CI, Shapley RM (2009) Spatial spread of the local field potential and its laminar variation in visual cortex. J Neurosci 29:11540-11549.

Zanos TP, Mineault PJ, Pack CC (2011) Removal of spurious correlations between spikes and local field potentials. J Neurophysiol 105:474-486.

Zhou Z, Bernard MR, Bonds AB (2008) Deconstruction of spatial integrity in visual stimulus detected by modulation of synchronized activity in cat visual cortex. J Neurosci 28:3759-3768. 